\title{
Multilevel Decision-Making: A Survey
}

\author{
Jie Lu ${ }^{\mathrm{a}}$, Jialin $\mathrm{Han}^{\mathrm{a}, \mathrm{b}}$, Yaoguang $\mathrm{Hu}^{\mathrm{b}}$, Guangquan Zhang ${ }^{\mathrm{a}}$ \\ ${ }^{\mathrm{a}}$ Decision Systems and e-Service Intelligence Laboratory, Centre for Quantum Computation and \\ Intelligent Systems, Faculty of Engineering and Information Technology, \\ University of Technology Sydney, Australia \\ ${ }^{\mathrm{b}}$ Industrial and Systems Engineering Laboratory, School of Mechanical Engineering, \\ Beijing Institute of Technology, China
}
E-mail: Jie.Lu@uts.edu.au (J. Lu), Jialin.Han@student.uts.edu.au (J. Han), hyg@bit.edu.cn (Y.Hu), Guangquan.Zhang@uts.edu.au (G. Zhang).

\begin{abstract}
Multilevel decision-making techniques aim to deal with decentralized management problems that feature interactive decision entities distributed throughout a multiple level hierarchy. Significant efforts have been devoted to understanding the fundamental concepts and developing diverse solution algorithms associated with multilevel decision-making by researchers in areas of both mathematics/computer science and business areas. Researchers have emphasized the importance of developing a range of multilevel decision-making techniques to handle a wide variety of management and optimization problems in real-world applications, and have successfully gained experience in this area. It is thus vital that a high quality, instructive review of current trends should be conducted, not only of the theoretical research results but also the practical developments in multilevel decision-making in business. This paper systematically reviews up-to-date multilevel decision-making techniques and clusters related technique developments into four main categories: bi-level decision-making (including multi-objective and multi-follower situations), tri-level decision-making, fuzzy multilevel decision-making, and the applications of these techniques in different domains. By providing state-of-the-art knowledge, this survey will directly support researchers and practical professionals in their understanding of developments in theoretical research results and applications in relation to multilevel decision-making techniques.
\end{abstract}

Keywords: Multilevel decision-making, bi-level programming, fuzzy decision-making.

\section{Introduction}

Multilevel decision-making techniques, motivated by Stackelberg game theory [147] and presented by multilevel mathematical programming, have been developed to address compromises between the interactive decision entities that are distributed throughout a hierarchical organization. In a multilevel decision-making process, decision entities at the upper level and the lower level are 
respectively termed the leader and the follower, and make their individual decisions in sequence with the aim of optimizing their respective objectives. This decision-making process means that the leader has priority in making its own decision and the follower reacts after and in full knowledge of the leader's decision; however, the leader's decision is implicitly affected by the follower's reaction. Since this category of hierarchical decision-making often appears in many decentralized management problems in the real world, multilevel decision-making has motivated a number of researches on decision models [101, 103], solution approaches [126] and applications [89].

The original appearance of multilevel decision-making can be traced back to 1973, in a paper authored by Bracken and McGill [25], although a wide range of related research has been undertaken since the 1980s under the following designations: multilevel programming, multilevel optimization and multilevel decision-making. Early efforts in multilevel decision-making were primarily devoted to addressing optimality conditions and solution algorithms for solving basic linear, nonlinear and discrete problems, in which only one decision entity is involved at each decision level with the aim of optimizing a unique objective. Although the multilevel decision-making problem has been proved to be NP-hard by Ben-Aved and Blair [21] and Bard [16], many methods/algorithms have been developed for solving typical cases in bi-level decision-making problems, such as extreme point algorithms, branch-and-bound algorithms, complementary pivot algorithms, descent methods and penalty function methods [17, 45]. These methods/algorithms can be considered to be traditional solution approaches for solving multilevel (mainly bi-level) decision-making problems.

From a practical point of view, there are two fundamental issues in supporting a multilevel decision-making process: one is how to develop a multilevel decision model to describe a hierarchical decision-making process, and the other is how to find an optimal solution to the resulting decision model. For the first issue, bi-level decision-making models with multiple optima, involving multiple objectives, multiple leaders and multiple followers, have been proposed to handle different characteristics at different decision levels. Tri-level decision-making, another typical multilevel decision form that is more complex than bi-level decision-making, has been well studied in model establishment. For the second issue, a set of solution approaches has been developed to solve these models. In the meanwhile, attention has been paid to uncertain issues in both model parameter determination and solution process, and fuzzy set technique has been used in both multilevel decision modeling and solution approaches. 
Multilevel decision problems have recently increasingly appeared in decentralized management situations in the real world and have become highly complicated and large-scale, particularly with the development of economic integration and in the current age of big data; for example, business firms nowadays usually work in a decentralized manner in a complex commercial network comprised of suppliers, manufacturers, sales and logistics companies, customers and other specialized service functions [75]. Therefore, to help researchers understand the development experience of multilevel decision-making techniques and to assist practitioners to handle related decision-making problems in practice, this paper reviews the latest research on multilevel decision-making involving theoretical research results and applications, which are clustered into four categories: bi-level decision-making, tri-level decision-making, fuzzy multilevel decision-making, and applications of multilevel decision-making techniques.

Several survey papers $[22,39,40,89,126,154]$ on multilevel programming/decision-making have been published in the last 20 years. However, these papers focus on early research on basic bi-level decision-making, either with traditional solution approaches only or a specific domain of applications. For example, the survey papers by Ben-Ayed [22], Vicente and Calamai [154] and Colson, et al. [39, 40] presented overviews of bi-level programming, which only focused on traditional solution concepts and approaches for solving basic bi-level decision problems. Sakawa and Nishizaki [126] reviewed interactive fuzzy programming approaches for solving bi-level and multilevel decision problems. Kalashnikov, et al. [89] surveyed bi-level decision-making techniques on a specific application domain of energy networks. Although these survey papers have provided good references for researchers, there is an urgent need for an updated and more comprehensive review of recent developments in multilevel decision-making techniques. More importantly, in contrast to these papers that are limited to solution approaches for solving bi-level mathematical programming, this survey focuses on a variety of decision models, solution approaches and application domains from the perspective of different categories of multilevel decision-making.

To conduct this literature review, three main types of article are carefully reviewed: Type1 articles on bi-level programming/decision-making; Type 2 - articles on tri-level programming/decision-making and fuzzy multilevel programming/decision-making; and Type 3 articles on multilevel decision-making applications. The search and selection of these articles were performed according to the following five steps:

Step 1. Publication database identification and determination. Publication databases, such as 
Science Direct, ACM Digital Library, IEEE Xplore and SpringerLink, were selected to provide a comprehensive bibliography of papers on multilevel decision-making.

Step 2. Preliminary screening of articles. The search was first performed based on related keywords of multilevel decision-making. The articles were then selected as references if they satisfied one of the following criteria that they (1) proposed decision models related to multilevel decision-making; (2) developed solution concepts and approaches for solving multilevel decision problems; (3) provided a real-world multilevel decision-making application. Step 3. Result filtering for Type 1 articles. Based on the keywords related to bi-level decision-making, these papers were divided into three groups using "topic clustering": (a) basic bi-level decision-making involving linear, nonlinear and discrete problems; (b) bi-level multi-objective decision-making; and (c) bi-level multi-leader and/or multi-follower decision-making. These papers were selected according to three criteria: (1) novelty - published within the last decade; (2) impact - published in high quality (high impact factor) journals, or in conference proceedings or book chapters but with high citations; and (3) typicality - only the most typical methodologies were retained. These papers are mainly used in Section 2.

Step 4. Type 2 article selection. The techniques in relation to tri-level and multilevel decision-making, and fuzzy multilevel decision-making were analyzed and the related papers were selected according to the three criteria: (1) novelty, (2) impact, and (3) typicality. For this category of technique, relevant decision models and solution approaches were carefully reviewed. These papers are mainly used in Sections 3 and 4.

Step 5. Result filtering for Type 3 articles. Based on the keywords related to multilevel decision-making applications, these papers were divided into four groups using "topic clustering": (a) supply chain management; (b) traffic and transportation; (c) energy management, and (d) safety and accident management. These papers were selected according to the four criteria: (1) novelty; (2) impact; (3) coverage - reported a new or particular application domain; and (4) typicality - only the most typical examples related to similar applications were retained. These papers aim to introduce the applications of multilevel decision-making techniques, which are mainly used in Section 5.

Ultimately, 179 articles in total were selected as the final reference list for this paper.

The main contributions of this paper are: (1) the paper comprehensively and perceptively summarizes research achievements on multilevel decision-making techniques from the point of 
view of theoretical research results and applications, and strategically clusters the multilevel decision-making techniques into four categories; (2) for each multilevel decision-making technique, it carefully analyzes typical decision models and effectively identifies the specific requirements for solution approaches in the category. This will directly motivate and support researchers and practitioners to promote the popularization and application of multilevel decision-making techniques in different domains; (3) it provides several methods for conducting the set of available multilevel decision-making test problems and covers very new multilevel decision-making technique applications; and (4) it particularly suggests several very innovative emerging research topics/directions in the area of multilevel decision-making.

The remainder of this paper is structured as follows. In Section 2, the bi-level decision-making models and solution approaches are reviewed and analyzed. Section 3 presents the tri-level decision-making models and solution approaches. Section 4 addresses fuzzy multilevel (including bi-level and tri-level) decision-making techniques. Section 5 discusses the principal applications of multilevel decision-making techniques. Section 6 presents our comprehensive analysis and prospective research topics.

\section{Bi-level decision-making}

This section first reviews the development of techniques for solving basic bi-level decision-making problems. It then addresses the developments of bi-level decision-making with multiple optima involving bi-level multi-objective decision-making, bi-level multi-leader decision-making and bi-level multi-follower decision-making.

\subsection{Basic bi-level decision-making}

Basic bi-level decision-making, as found in a bi-level programming situation, has only one decision entity attempting to optimize a unique objective at each decision level. The general formulation for basic bi-level decision-making is described by a bi-level program as Definition 1 .

Definition 1 [17] For $x \in X \subset R^{p}, y \in Y \subset R^{q}$, a general bi-level decision problem is defined as:

$$
\begin{aligned}
& \min _{x \in X} F(x, y) \quad \text { (1st level) } \\
& \text { s.t. } G(x, y) \leq 0,
\end{aligned}
$$

where, for each $x$ given by the 1st level, $y$ solves 


$$
\min _{y \in Y} f(x, y) \quad \text { (2nd level) }
$$

s.t. $g(x, y) \leq 0$,

where $x, y$ are the decision variables of the first level and the second level respectively; $F, f: R^{p} \times R^{q} \rightarrow R^{1}$ are the objective functions of the first level and the second level respectively; $G: R^{p} \times R^{q} \rightarrow R^{m}, g: R^{p} \times R^{q} \rightarrow R^{n}$ are the constraint conditions of the first level and the second level respectively. The sets $X$ and $Y$ place additional restrictions on the decision variables, such as upper and lower bounds or integrality requirements [17].

Relevant solution concepts in relation to bi-level decision problem (1) are defined as follows.

\section{Definition 2 [17]}

1) The constraint region of the bi-level decision problem:

$$
S=\{(x, y) \in X \times Y: G(x, y) \leq 0, g(x, y) \leq 0\} .
$$

2) The feasible set of the second level for each fixed $x$ :

$$
S(x)=\{y \in Y: g(x, y) \leq 0\} .
$$

3) The rational reaction set of the second level:

$$
P(x)=\{y \in Y: y \in \operatorname{ar} \operatorname{gni~n} f[(x, y): y \in S(x)] .
$$

4) The inducible region of the bi-level decision problem:

$$
I R=\{(x, y):(x, y) \in S, y \in P(x)\}
$$

5) The optimal solution set of the bi-level decision problem:

$$
O S=\{(x, y):(x, y) \in \arg \min [F(x, y):(x, y) \in I R]\} .
$$

It is clear from Definition 2 that the constraint domain associated with a bi-level decision problem is implicitly determined by two optimization problems that must be solved in a predetermined sequence from the first level to the second level. The bi-level decision problem (1) is equivalent to optimizing the leader's objective $F$ over the inducible region $I R$.

Although a number of methodologies have been developed for solving basic bi-level decision problems involving linear, nonlinear and discrete versions, the main pursuit of this section is to summarize the related solution algorithms developed in the last decade, because the basic concepts, complexity, optimality conditions and traditional algorithms have been carefully reviewed in the existing surveys [22, 39, 40, 154]. 


\subsubsection{Linear bi-level decision-making}

Definition 3 [17] Based on Definition 1, for $x \in X \subset R^{p}, y \in Y \subset R^{q}$, and $F, f: R^{p} \times R^{q} \rightarrow R^{1}$, the linear bi-level decision problem can be written as follows:

$$
\begin{aligned}
& \min _{x \in X} F(x, y)=c_{1} x+d_{1} y \quad \text { (1st level) } \\
& \text { s.t. } A_{1} x+B_{1} y \leq b_{1}
\end{aligned}
$$

where, for each $x$ given by the 1st level, $y$ solves

$$
\begin{array}{ll}
\min _{y \in Y} f(x, y)=c_{2} x+d_{2} y & \text { (2nd level) } \\
\text { s.t. } & A_{2} x+B_{2} y \leq b_{2},
\end{array}
$$

where $c_{1}, c_{2} \in R^{p}, d_{1}, d_{2} \in R^{q}, b_{1} \in R^{m}, b_{2} \in R^{n}, A_{1} \in R^{m \times p}, B_{1} \in R^{m \times q}, A_{2} \in R^{n \times p}, B_{2} \in R^{n \times q}$. In accordance with Definition 2, readers can easily elicit the related solution concepts for linear bi-level decision problem (2). Note that we only consider continuous decision variables in this section.

In terms of solving linear bi-level decision problems, the traditional algorithms can be classified into three main categories: the vertex enumeration approaches [23, 35, 135, 152] based on an important characteristic of bi-level programming whereby an optimal solution occurs at a vertex of the constraint region; the Kuhn-Tucker approaches involving branch-and-bound algorithms [18, 19, $60,136]$ and complementary pivot algorithms [23, 85, 115], in which the upper-level problem includes the lower-level's optimality conditions as extra constraints; and the penalty function approaches $[6,163]$ which append a penalty term of the lower-level problem to the objective function of the upper-level problem.

In recent years, Audet, et al. [11] proposed a disjunctive cuts method for a linear bi-level decision problem with continuous variables. Audet, et al. [12] considered the equivalences between linear mixed 0-1 integer programming problems and linear bi-level decision problems, and proposed a finite and exact branch-and-cut algorithm for solving such problems. Glackin, et al. [71] addressed the relationship between linear multi-objective programs and linear bi-level programs and presented an algorithm for solving linear bi-level programs that uses simplex pivots on an expanded tableau. Calvete and Galé [30] addressed linear bi-level programs in which the coefficients of both objective functions are interval numbers and developed two algorithms based on ranking extreme points to solve such problems. Ren and Wang [121] proposed a cutting plane method to solve the 
linear bi-level decision problem with interval coefficients in both objective functions.

The aforementioned approaches provide ways to solve linear bi-level decision problems. However, these approaches are very time-consuming when solving large-scale problems; for example, the computational load of the $K$ th-Best algorithm will increase steeply with the increase in the mass of variables and constraints. To overcome this issue, a range of heuristic algorithms have been developed to solve bi-level decision problems. Gendreau, et al. [70] used an adaptive search method related to the tabu search meta-heuristic to solve the linear bi-level decision problem. Hejazi, et al. [82] proposed a method based on genetic algorithm for solving linear bi-level decision problems. Calvete, et al. [33] developed a genetic algorithm for solving a class of linear bi-level decision problems in which both objective functions are linear and the common constraint region is a polyhedron. $\mathrm{Hu}$, et al. [84] presented a neural network approach for solving linear bi-level decision problems. Lan, et al. [96] proposed a hybrid algorithm that combines neural network and tabu search for solving linear bi-level decision problems. Kuo and Huang [94] developed a particle swarm optimization (PSO) algorithm with swarm intelligence to solve linear bi-level decision problems. In addition, Calvete, et al. [33] presented a method for the test set construction of linear bi-level decision problems especially for generating large-scale problems, which can be employed to assess the efficiency performance of related algorithms.

\subsubsection{Nonlinear bi-level decision-making}

With respect to Definition 1 , if the objective functions $F(x, y), f(x, y)$ or the constraint conditions $G(x, y) \leq 0, g(x, y) \leq 0$ are nonlinear formulations, the bi-level program is known as a nonlinear bi-level decision problem, which is much more difficult to solve than linear versions.

In early research in solving nonlinear bi-level decision problems, Bard [14] extended the traditional branch-and-bound algorithm to solve nonlinear convex bi-level decision problems. Edmunds and Bard [51] used a branch-and-bound algorithm and a cutting-plane algorithm to solve various versions of nonlinear bi-level decision problems when certain convexity conditions hold. Al-Khayyal, et al. [1] developed a branch-and-bound algorithm and a piecewise linear approximation method to find the global minimum for a class of nonlinear bi-level decision problems based on an equivalent system of convex and separable quadratic constraints. Vicente and Calamai [154] introduced two descent methods for a special instance of bi-level programs where the second-level problem is strictly convex quadratic. 
In recent years, Tuy, et al. [151] showed that a nonlinear bi-level decision problem can be transformed into a monotonic optimization problem which can then be solved by a branch-reduce-and-bound method using monotonicity cuts. Mersha and Dempe [109] studied the application of a class of direct search methods and solved bi-level decision problems containing convex lower level problems with strongly stable optimal solutions. Mitsos, et al. [111] presented a bounding algorithm for the global solution of nonlinear bi-level programs involving nonconvex objective functions in both decision levels.

In regard to related heuristic algorithms, Wang, et al. [159] transformed a special nonlinear bi-level decision problem into an equivalent single objective nonlinear programming problem that can be solved by an evolutionary algorithm. Wan, et al. [157] presented a hybrid intelligent algorithm of PSO and chaos searching technique for solving nonlinear bi-level decision problems. Wan, et al. [156] also developed a novel evolutionary algorithm, called the estimation of distribution algorithm, for solving a special class of nonlinear bi-level decision problems in which the lower-level problem is a convex program for each given upper-level decision. Lv et al. [106, 107] and He, et al. [81] proposed neural network methods for solving nonlinear bi-level decision problems. It is notable that Sinha, et al. [141] proposed a procedure for designing the test set of nonlinear bi-level decision problems and presented the corresponding computational results for these test problems using a nested bi-level evolutionary algorithm. Researchers can consider these test problems as the benchmark for examining the effectiveness of their own algorithms.

\subsubsection{Discrete bi-level decision-making}

In many bi-level decision-making problems, a subset of the variables is restricted to take on discrete values [17]. A problem can be considered to be a general discrete bi-level decision problem when the decision variables in Definition 1 are discrete, e.g. integer programming. Clearly, the linear bi-level decision problem (2) will become a discrete linear bi-level program if the decision variables are discrete rather than continuous.

Discrete variables can complicate bi-level decision problems by several orders of magnitude and render all but the smallest instances unsolvable [17]. Bard and Moore [20], Moore and Bard [112] and Wen and Yang [161] therefore proposed traditional branch-and-bound algorithms for finding solutions to integer linear bi-level decision-making problems. Vicente, et al. [155] designed penalty function methods for solving discrete linear bi-level decision problems. Edmunds and Bard [50] 
developed a branch-and-bound algorithm to solve a mixed-integer nonlinear bi-level decision problem.

Recently, Köppe, et al. [87] proposed a parametric integer programming algorithm for solving a mixed integer linear bi-level decision problem where the follower solves an integer program with a fixed number of variables. $\mathrm{Xu}$ and Wang [165] solved a mixed integer linear bi-level decision problem using an exact algorithm. The algorithm relies on three simplifying assumptions, explicitly considers finite optimal, infeasible and unbounded cases, and is proved to terminate finitely and correctly. Domínguez and Pistikopoulos [49] addressed two algorithms using multiparametric programming techniques respectively for solving two categories of integer bi-level decision problems: one category consists of pure integer problems where integer variables of the first level appear in the linear or polynomial problem of the second level, and the other consists of mixed-integer problems where integer and continuous variables of the first level appear in the linear or polynomial problem of the second level. Sharma, et al. [134] discussed an integer bi-level decision problem with bounded variables in which the objective function of the first level is linear fractional, the objective function of the second level is linear and the common constraint region is a polyhedron. They proposed an iterative algorithm to find an optimal solution to the problem. Faísca, et al. [55] proposed a global optimization approach to solve quadratic bi-level and mixed integer linear bi-level problems, with or without right-hand-side uncertainty. Mitsos [110] presented an algorithm based on the research by Mitsos, et al. [111] for the global optimization of nonlinear bi-level mixed-integer programs, which relies on a convergent lower bound and an optional upper bound.

In relation to heuristic algorithms for solving discrete bi-level decision problems, Wen and Huang [160] reported a mixed-integer linear bi-level decision-making formulation in which zero-one decision variables are controlled by the first level and real-value decision variables are controlled by the second level. An algorithm based on the short term memory component of tabu search, called simple tabu search, was developed to solve the problem. Nishizaki and Sakawa [114] presented a method using genetic algorithms for obtaining optimal solutions to integer linear bi-level decision problems.

\subsection{Bi-level multi-objective decision-making}

When multiple conflicting objectives for each decision entity exist in a bi-level decision 
problem, this is known as a bi-level multi-objective (BLMO) decision problem.

Definition 4 [42] For $x \in X \subset R^{p}, y \in Y \subset R^{q}$, a general BLMO decision problem is formulated as:

$$
\begin{aligned}
& \min _{x \in X} F(x, y)=\left(F_{1}(x, y), F_{2}(x, y), \ldots, F_{M}(x, y)\right) \quad \text { (1st level) } \\
& \text { s.t. } G(x, y) \leq 0
\end{aligned}
$$

where, for each $x$ given by the 1st level, $y$ solves

$$
\begin{aligned}
& \min _{y \in Y} f(x, y)=\left(f_{1}(x, y), f_{2}(x, y), \ldots, f_{N}(x, y)\right) \quad \text { (2nd level) } \\
& \text { s.t. } g(x, y) \leq 0
\end{aligned}
$$

where $x, y$ are the decision variables of the first level and the second level respectively; $F_{i}, f_{j}: R^{p} \times R^{q} \rightarrow R^{1}, i=1,2, \ldots, M, j=1,2, \ldots, N$ are the conflicting objective functions of the first level and the second level respectively; $G: R^{p} \times R^{q} \rightarrow R^{m}, g: R^{p} \times R^{q} \rightarrow R^{n}$ are the constraint conditions of the first level and the second level respectively. The sets $X$ and $Y$ place additional restrictions on the decision variables, such as upper and lower bounds or integrality requirements. It is notable that Definition 2 also applies to the BLMO decision problem (3).

Many algorithms have been developed to solve bi-level multi-objective (BLMO) decision problems in various versions. Ankhili and Mansouri [8] addressed a class of linear bi-level programs where the upper level is a linear scalar optimization problem and the lower level is a linear multi-objective optimization problem; they approached the problems via an exact penalty method. Calvete and Galé [28] presented a number of methods of computing efficient solutions to solve linear bi-level decision problems with multiple objectives at the upper level; all the methods result in solving linear bi-level problems with a single objective function at each level based on both weighted sum scalarization and scalarization techniques. Emam [53] proposed an interactive approach for solving bi-level integer fractional multi-objective decision problems. Eichfelder [52] discussed a nonlinear nonconvex BLMO decision problem using an optimistic approach in which the feasible points of the upper-level objective function can be expressed as the set of minimal solutions of a single-level multi-objective optimization problem. The BLMO decision problem is then solved by an iterative process, again using sensitivity theorems.

From the aspect of using heuristic algorithms for solving BLMO decision problems, Alves and Costa [3] presented an improved PSO algorithm to solve linear bi-level decision problems with 
multiple objectives at the upper level. Calvete and Galé [29] developed an exact algorithm and a metaheuristic algorithm to solve linear bi-level decision problems with multiple objectives at the lower level. Zhang, et al. [179] proposed a hybrid PSO algorithm with crossover operator to solve high dimensional bi-level multi-objective decision problems. Deb and Sinha [42] proposed a viable and hybrid evolutionary-cum-local-search based algorithm for solving BLMO decision problems. Note that Deb and Sinha [41] also presented a method for constructing the test set of BLMO decision problems.

\subsection{Bi-level multi-leader and/or multi-follower decision-making}

In a bi-level decision problem, multiple decision entities may exist at each level, and this is known as a bi-level multi-leader and/or multi-follower decision problem. A general bi-level multi-leader (BLML) decision problem can be defined as Definition 5.

Definition 5 [175] For $x_{i} \in X_{i} \subset R^{p_{i}}, y \in Y \subset R^{q}, i=1,2, \ldots, L$, a general BLML decision problem in which $L$ leaders and one follower are involved can be described as:

$$
\begin{aligned}
& \min _{x_{i} \in X_{i}} F_{i}(x, y) \quad \text { (1st level) } \\
& \text { s.t. } G_{i}(x, y) \leq 0
\end{aligned}
$$

where, for each $x$ given by the 1st level, $y$ solves

$$
\begin{aligned}
& \min _{y \in Y} f(x, y) \quad \text { (2nd level) } \\
& \text { s.t. } g(x, y) \leq 0
\end{aligned}
$$

where $x=\left(x_{1}, x_{2}, \ldots, x_{L}\right), x_{i}$ and $y$ are the decision variables of the $i$ th leader and the follower respectively; $F_{i}, f: R^{p_{1}} \times \ldots \times R^{p_{L}} \times R^{q} \rightarrow R^{1}$ are the objective functions of the $i$ th leader and the follower respectively; $\quad G_{i}: R^{p_{1}} \times \ldots \times R^{p_{L}} \times R^{q} \rightarrow R^{m_{i}} \quad, \quad g: R^{p_{1}} \times \ldots \times R^{p_{L}} \times R^{q} \rightarrow R^{n}$ are the constraint conditions of the $i$ th leader and the follower respectively. The sets $X$ and $Y$ place additional restrictions on the decision variables, such as upper and lower bounds or integrality requirements. It is clear in Definition 5 that, when leaders make their individual decisions, they need to not only take into account the implicit reaction of the follower but also consider the decision results given by their counterparts at the first level.

In relation to research on bi-level multi-leader decision-making, DeMiguel and Huifu [44] studied a stochastic BLML decision model and proposed a computational approach to find a 
Stochastic Multiple-leader Stackelberg-Nash-Cournot (SMS) equilibrium based on the sample average approximation method. Zhang, et al. [175] introduced a framework for the bi-level multi-leader (BLML) decision problem, in which they presented different BLML decision models in line with various relationships between multiple leaders. The authors also proposed a PSO algorithm to find a solution for BLML decision problems based on the related solution concepts.

In contrast to the limited discussion on BLML decision-making, researchers have paid considerably more attention to bi-level multi-follower (BLMF) decision-making. A general BLMF decision problem in which one leader and $k$ followers are involved can be defined as Definition 6 .

Definition 6 [175] For $x \in X \subset R^{p}, y_{i} \in Y_{i} \subset R^{q_{i}}, i=1,2, \ldots, k$, a general BLMF decision problem in which one leader and $k$ followers are involved can be written as:

$$
\begin{aligned}
& \min _{x \in X} F(x, y) \quad \text { (1st level) } \\
& \text { s.t. } G(x, y) \leq 0
\end{aligned}
$$

where, for each $x$ given by the 1st level, $y_{i}$ solves the $i$ th follower's problem

$$
\begin{aligned}
& \min _{y_{i} \in Y_{i}} f_{i}(x, y) \quad \text { (2nd level) } \\
& \text { s.t. } g_{i}(x, y) \leq 0
\end{aligned}
$$

where $y=\left(y_{1}, y_{2}, \ldots, y_{k}\right), x$ and $y_{i}$ are the decision variables of the leader and the $i$ th follower respectively; $F, f_{i}: R^{p} \times R^{q_{1}} \times \ldots \times R^{q_{k}} \rightarrow R^{1}$ are the objective functions of the leader and the $i$ th follower respectively; $\quad G: R^{p} \times R^{q_{1}} \times \ldots \times R^{q_{k}} \rightarrow R^{m} \quad, \quad g_{i}: R^{p} \times R^{q_{1}} \times \ldots \times R^{q_{L}} \rightarrow R^{n_{i}}$ are the constraint conditions of the leader and the $i$ th follower respectively. The sets $X$ and $Y$ place additional restrictions on the decision variables, such as upper and lower bounds or integrality requirements. It can be seen in Definition 6 that followers need to consider the decision results of their counterparts as references when making their individual decisions in view of the decision given by the leader.

Anandalingam and Apprey [5] first presented a linear BLMF decision model, known as a linear bi-level multi-agent system, and developed a penalty function approach to solve the problem. Liu [98] designed a genetic algorithm for solving Stackelberg-Nash equilibrium of nonlinear BLMF decision problems in which there might be an information exchange between the followers. Based on previous research, Lu, et al. [101] proposed a general framework of BLMF decision-making that considers three main relationships between multiple followers: the uncooperative relationship, the 
referential-uncooperative relationship, and the partial-cooperative relationship. The research on BLMF decision-making after $\mathrm{Lu}$, et al. [101] was structured on the general framework. Calvete and Galé [27] subsequently presented a approach for solving the linear BLMF decision problem with uncooperative followers, which converted the BLMF problem to a bi-level problem with one leader and one follower. Shi et al. [137, 138] extended the Kth-best algorithm to solve linear BLMF decision problems with uncooperative and partial-cooperative relationships respectively between followers. Lu, et al. [102] and Lu and Shi [100] respectively adopted the extended Kuhn-Tucker algorithm and the extended branch-and-bound algorithm to solve the referential-uncooperative linear BLMF decision problem.

Nie [113] developed and characterized discrete-time dynamic bi-level multi-leader and multi-follower (BLMLMF) games with leaders in turn, and a dynamic programming algorithm was employed to solve this problem. Gao [63] developed PSO-based algorithms to solve BLML, BLMF and BLMLMF decision problems. Sinha, et al. [142] used a computationally intensive nested evolutionary algorithm to find an optimal solution for a multi-period BLMLMF decision problem with nonlinear and discrete variables.

To conclude, a number of solution approaches involving exact algorithms and heuristic algorithms have been developed for solving a variety of bi-level decision problems. Nevertheless, these algorithms are still time-consuming for solving large-scale bi-level decision problems in nonlinear, discrete and multi-optima versions. Also, it is difficult and sometimes almost impossible to extend these algorithms to solve tri-level and multilevel decision problems. Therefore, it is necessary to develop more effective algorithms for solving large-scale bi-level problems, which can be also extended to solve tri-level and multilevel decision problems.

\section{Tri-level decision-making}

Decentralized decision-making problems within a hierarchical system are often comprised of more than two levels in many applications, which is known as tri-level and multilevel decision-making.

Definition 7 [56] For $x \in X \subset R^{p}, y \in Y \subset R^{q}, z \in Z \subset R^{r}$, a general tri-level decision problem is defined as:

$$
\begin{aligned}
& \min _{x \in X} f_{1}(x, y, z) \quad \text { (1st level) } \\
& \text { s.t. } g_{1}(x, y, z) \leq 0
\end{aligned}
$$


where, for each $x$ given by the 1 st level, $(y, z)$ solve the problems of the 2 nd and 3rd levels:

$$
\min _{y \in Y} f_{2}(x, y, z) \quad \text { (2nd level) }
$$

s.t. $g_{2}(x, y, z) \leq 0$,

where, for each $(x, y)$ given by the 1 st and 2 nd levels, $z$ solves

$$
\min _{z \in Z} f_{3}(x, y, z) \quad \text { (3rd level) }
$$

s.t. $g_{3}(x, y, z) \leq 0$,

where $x, y, z$ are the decision variables of the three levels respectively; $f_{1}, f_{2}, f_{3}: R^{p} \times R^{q} \times R^{r} \rightarrow R^{1}$ are the objective functions of the three levels respectively; $g_{i}: R^{p} \times R^{q} \times R^{r} \rightarrow R^{k_{i}}, i=1,2,3$ are the constraint conditions of the three levels respectively.

Relevant solution concepts are proposed as follows based on the nested hierarchical structure of tri-level decision-making.

\section{Definition 8 [56]}

1) The constraint region of the tri-level decision problem:

$$
S=\left\{(x, y, z) \in X \times Y \times Z: g_{i}(x, y, z) \leq 0, i=1,2,3\right\} .
$$

2) The feasible set of the second level for each fixed $x$ :

$$
S(x)=\left\{(y, z) \in Y \times Z: g_{2}(x, y, z) \leq 0, g_{3}(x, y, z) \leq 0\right\} .
$$

3) The feasible set of the third level for each fixed $(x, y)$ :

$$
S(x, y)=\left\{z \in Z: g_{3}(x, y, z) \leq 0\right\} .
$$

4) The rational reaction set of the third level:

$$
P(x, y)=\left\{z \in Z: z \in \text { ar gn i nff } f_{3}(x, y, z): z \in S(x, y)\right] .
$$

5) The rational reaction set of the second level:

$$
P(x)=\left\{(y, z) \in Y \times Z:(y, z) \in \arg \min \left[f_{2}(x, y, z):(y, z) \in S(x), z \in P(x, y)\right] .\right.
$$

6) The inducible region of the tri-level decision problem:

$$
I R=\{(x, y, z):(x, y, z) \in S,(y, z) \in P(x)\} .
$$

7) The optimal solution set of the tri-level decision problem:

$$
O S=\left\{(x, y, z):(x, y, z) \in \arg \min \left[f_{1}(x, y, z):(x, y, z) \in I R\right]\right\} .
$$

While the majority of studies on multilevel decision-making have focused on bi-level 
decision-making, research on tri-level decision problems has increasingly attracted investigations into solution approaches since tri-level decision-making can be applied to handle many decentralized decision problems in the real world [103]. Bard [15] first presented an investigation into linear tri-level decision-making and designed a cutting plane algorithm to solve such problems, based on which White [162] proposed a penalty function approach for linear tri-level decision problems. Anandalingam [4] and Sinha [143] developed Kuhn-Tucker transformation methods to find local optimal solutions for linear tri-level decision problems. Ruan, et al. [124] discussed the optimality conditions and related geometric properties of a linear tri-level decision problem with dominated objective functions. Faísca, et al. [56] studied a multiparametric programming approach to solve tri-level hierarchical and decentralized optimization problems based on parametric global optimization for bi-level decision-making [55]. Zhang, et al. [176] developed a tri-level Kth-Best algorithm to solve linear tri-level decision problems. In addition, Han, et al. [77] presented a PSO algorithm for solving linear and nonlinear tri-level decision problems.

A category of approaches based on fuzzy programming has been also developed to solve multilevel decision problems involving bi-level and tri-level programs. Lai [95] first proposed a fuzzy approach to find a satisfactory solution to the linear multilevel decision problem using concepts of membership functions of individual optimality and the satisfactory degree of individual decision power. Shih, et al. [139] extended Lai's concepts and adopted tolerance membership functions and multiple objective optimization to develop a fuzzy approach for solving the above problems. Sakawa, et al. [128] presented an interactive fuzzy programming approach for linear multilevel decision problems by updating the satisfactory degrees of decision entities at the upper level with considerations of overall satisfactory balance between all levels. Their interactive fuzzy programming approach overcomes the inconsistency between the fuzzy goals of objectives and decision variables that existed in the research developed by Lai [95] and Shih, et al. [139]. Sinha $[144,145]$ developed an alternative multilevel decision technique based on fuzzy mathematical programming, which considered a sequential order of the multilevel hierarchy and took into account the preference of the decision entity at each level. Pramanik and Roy [119] and Arora and Gupta [10] each proposed a fuzzy goal programming approach to solve linear multilevel decision problems using definitions of tolerance membership functions and satisfactory degree of decision entities.

To solve tri-level decision-making problems with multiple optima, Shih, et al. [139] proposed a 
tri-level decision model with multiple followers and developed a fuzzy approach to solve the model. $\mathrm{Lu}$, et al. [103] presented a framework for tri-level multi-follower (TLMF) decision-making research and developed 64 standard situations to describe various relationships among multiple followers. Based on the TLMF framework, Han et al. [75, 76, 78] discussed related solution concepts and extended the bi-level Kth-Best algorithm to solve three categories of linear TLMF decision problems with cooperative, uncooperative and reference-based uncooperative relationships respectively between multiple followers at the same level.

In summary, although these approaches provide ways to find solutions to tri-level decision problems, there are three noticeable drawbacks to adopting these approaches to solve tri-level decision problems. First, the existing approaches are limited to solving tri-level decision problems in linear format or in a special situation where all decision entities from different levels share the same constraint conditions and prefer to cooperate with one another. Second, the fuzzy approaches can only be used to find satisfactory solutions rather than optimal solutions, because cooperation is inhibited in classical multilevel decision-making problems, as has been commented on by Dempe [46]. Lastly, the efficiency of the proposed TLMF Kth-Best algorithm depends very much on the characteristics of the problem, that is, algorithmic performance may undergo a steeply downward trend in solving some TLMF decision problems. Consequently, further investigation into solution approaches for solving tri-level and multilevel decision problems is necessary.

\section{Fuzzy multilevel decision-making}

An important issue in modeling a multilevel decision problem is that the parameters involved are sometimes obtained through experiments or experts' understanding of the nature of the parameters. These parameters are often imprecisely or ambiguously known to the experts who establish the model, however, and clearly cannot be described by precise values [7]. Given this observation, it would certainly be more appropriate to interpret the experts' understanding of such parameters as fuzzy numerical data that can be represented by fuzzy sets theory. A multilevel decision problem in which the parameters are described by fuzzy values, often characterized by fuzzy numbers, is called a fuzzy multilevel decision problem $[170,175]$.

For the sake of simplicity, this section presents a general fuzzy linear bi-level decision problem based on Definition 3, described as Definition 9.

Definition 9 [169, 175] For $x \in X \subset R^{p}, y \in Y \subset R^{q}$, and $F, f: R^{p} \times R^{q} \rightarrow F(R)$, a general 
fuzzy linear bi-level decision problem can be written as follows:

$$
\begin{array}{ll}
\min _{x \in X} F(x, y)=\tilde{c}_{1} x+\tilde{d}_{1} y & \text { (1st level) } \\
\text { s.t. } & \tilde{A}_{1} x+\tilde{B}_{1} y \leq \tilde{b}_{1}
\end{array}
$$

where, for each $x$ given by the 1st level, $y$ solves

$$
\begin{array}{ll}
\min _{y \in Y} f(x, y)=\tilde{c}_{2} x+\tilde{d}_{2} y & \text { (2nd level) } \\
\text { s.t. } & \tilde{A}_{2} x+\widetilde{B}_{2} y \leq \tilde{b}_{2},
\end{array}
$$

where $\tilde{c}_{1}, \tilde{c}_{2} \in F^{p}(R), \quad \tilde{d}_{1}, \tilde{d}_{2} \in F^{q}(R), \quad \tilde{b}_{1} \in F^{m}(R), \quad \tilde{b}_{2} \in F^{n}(R), \quad \tilde{A}_{1} \in F^{m \times p}(R), \quad \tilde{B}_{1} \in F^{m \times q}(R)$, $\tilde{A}_{2} \in F^{n \times p}(R), \quad \tilde{B}_{2} \in F^{n \times q}(R), F(R)$ is the set of all finite fuzzy numbers.

Like multilevel decision-making under certainty, the majority of the research on fuzzy multilevel decision-making has focused on bi-level versions that have motivated numerous solution approaches [175]. Zhang and $\mathrm{Lu}$ [169] proposed a general fuzzy linear bi-level decision problem and developed an approximation Kuhn-Tucker approach to solve this problem. They also presented an approximation Kth-Best algorithm to solve the fuzzy linear bi-level decision problem [170]. Gao, et al. [65] proposed a programmable $\lambda$-cut approximation algorithm to solve a $\lambda$-cut set based fuzzy goal bi-level decision problem. Budnitzki [26] used the selection function approach and a modified version of the $K$ th-Best algorithm to solve a fuzzy linear bi-level decision problem. Sakawa, et al. [129] proposed an interactive fuzzy programming approach to find a satisfactory solution to a fuzzy linear bi-level decision problem. Pramanik [118] adopted a fuzzy goal programming approach to solve fuzzy linear bi-level decision problems.

Fuzzy bi-level decision-making with multiple optima has attracted numerous studies. Zhang, et al. [172] developed an approximation branch-and-bound algorithm to solve a fuzzy linear BLMO decision problem. Gao, et al. [67] proposed a $\lambda$-cut and goal-programming-based algorithm to solve fuzzy linear BLMO decision problems. Gao, et al. [64] focused on the fuzzy linear bi-level decision problem with multiple followers who share the common constraints and developed a PSO algorithm to solve the problem. Gao and Liu [62] integrated fuzzy simulation, neural network and genetic algorithm to produce a hybrid intelligent algorithm for solving a fuzzy nonlinear bi-level decision problem with multiple followers. Zhang, et al. [173] proposed a set of fuzzy linear bi-level multi-objective multi-follower (BLMOMF) decision models and developed an extended branch-and-bound algorithm to solve such problems. Zhang and $\mathrm{Lu}$ [171] developed an 
approximation Kth-Best algorithm to solve fuzzy linear BLMOMF decision problems with a cooperative relationship among multiple followers. Zhang, et al. [174] developed an approximation branch-and-bound algorithm to solve a fuzzy linear BLMOMF decision problem with a partial cooperative relationship among multiple followers.

In terms of the discussion about fuzzy tri-level and multilevel decision-making, Sakawa, et al. [129] extended their bi-level interactive fuzzy programming approach to solve fuzzy linear multilevel decision problems. They also extended the fuzzy approach to solve fuzzy linear multilevel fractional decision problems [130], fuzzy multilevel 0-1 decision problems [127] and fuzzy multilevel nonconvex decision problems [125]. Based on interactive fuzzy programming approaches, Osman, et al. [116] studied a fuzzy nonlinear tri-level decision problem with multiple objectives.

In general, these above solution approaches can be used to solve a range of fuzzy multilevel decision problems. However, these solution approaches are limited to handling special fuzzy numbers, e.g. triangular fuzzy numbers, or solving fuzzy decision problems in the linear version. In particular, these interactive fuzzy approaches can only solve fuzzy multilevel decision problems in which decision entities from different levels share the same constraint conditions and prefer to cooperate with one another. Under this special situation, the solution approaches aim to find satisfactory solutions rather than optimal solutions to fuzzy multilevel decision problems. Consequently, it is necessary to develop solution algorithms for solving much more general fuzzy multilevel decision problems.

\section{Applications of multilevel decision-making techniques}

Multilevel decision-making techniques have been widely applied to handle decentralized decision problems in the real world, in particular in the last five years. These applications largely fall into the following four areas: (1) supply chain management; (2) traffic and transportation network design; (3) energy management; and (4) safety and accident management.

\subsection{Supply chain management}

Supply chain management (SCM) requires decentralized decisions to be made at several stages in a complex hierarchical system which includes the location of business firms, the acquisition of raw materials, production planning and operations, inventory control, and the delivery and pricing of commodities. It is increasingly important to develop an efficient and easily-applicable 
decision-making methodology to handle conflict coordination and the decentralized nature of SCM $[97,131]$. In recognition of this, multilevel decision-making techniques have been applied to deal with many of the decentralized decision-making problems found in SCM.

Multilevel decision-making techniques in SCM have largely been applied to deal with the competitive location of facilities, production planning and operations, commodities distribution and pricing. With respect to the competitive location of facilities in SCM, Plastria and Vanhaverbeke [117] used discrete bi-level programs to adapt the competitive location model based on maximal covering to include the knowledge that a competitor will eventually enter the market with a single new facility. Küçükaydin, et al. [86] studied a problem in which a firm or franchise enters a market by locating new facilities near existing facilities belonging to a competitor and formulated the problem as a mixed-integer nonlinear bi-level decision model in which the firm entering the market is the leader and the competitor is the follower. Rider, et al. [123] presented a discrete bi-level decision model for determining optimal location and contract pricing of distributed generation in radial distribution systems where the upper-level optimization determines the allocation and contract prices of the distributed generation units, whereas the lower-level optimization models the reaction of the distribution company. Gang, et al. [61] proposed a bi-level multi-objective optimization model for a stone industrial park location problem with a hierarchical structure consisting of a local government and several stone enterprises in a random environment. The problem was solved using a bi-level interactive method based on a satisfactory solution and adaptive chaotic PSO.

For decentralized production planning and operations, Lukač, et al. [104] designed a mixed 0-1 integer bi-level decision model for a production planning problem with sequence dependent setups, in which the objective of the leader is to assign the products to the machines in order to minimize the total sequence dependent setup time, while the objective of the follower is to minimize the production, storage and setup cost of the machine. They developed a heuristic algorithm based on tabu search to solve the problem. Calvete, et al. [32] proposed a discrete bi-level program to model a hierarchical production-distribution planning problem in which two decision makers respectively controlling the production process and the distribution process do not cooperate because of different optimization strategies. An ant colony optimization approach was developed to solve the bi-level model. Kasemset and Kachitvichyanukul [90] presented a bi-level multi-objective mathematical model for a TOC (theory of constraints)-based job-shop scheduling problem and developed a PSO 
algorithm to solve the problem.

In terms of using multilevel decision-making techniques to handle commodities distribution and pricing problems in SCM, Gao, et al. [66] established two nonlinear bi-level pricing models for pricing problems between the vendor and the buyer, designated as the leader and the follower respectively, in a two-echelon supply chain. They developed a PSO-based algorithm to solve problems defined by these bi-level pricing models. Kuo and Han [93] applied linear bi-level programming to model a supply chain distribution problem and developed an efficient method based on a hybrid of the genetic algorithm and PSO algorithm to solve the resulting decision model. Kis and Kovács [91] presented a discrete bi-level decision model to handle an extension of the classical uncapacitated lot-sizing problem with backlogs, in which two autonomous and self-interested decision makers constitute a two-echelon supply chain. Qiu and Huang [120] presented a nonlinear bi-level decision model and an enumerative algorithm to describe and solve a SCM problem in which a supply hub in an industrial park and manufacturers interact to make their decisions on pricing, replenishment and delivery. Calvete, et al. [31] addressed a mixed integer bi-level optimization model for the planning of a decentralized distribution network consisting of manufacturing plants, depots and customers, and a metaheuristic approach based on evolutionary algorithms was developed to solve the optimization model. Ma, et al. [108] considered a two-echelon supply chain system with one manufacturer and one retailer, in which the manufacturer first purchases raw materials from the supplier; following production and processing by the manufacturer, the end products are sold to the retailer. By switching the leader and follower roles between the manufacturer and the retailer, the authors established two nonlinear bi-level decision models for joint pricing and lot-sizing and developed a PSO algorithm to solve the resulting models.

A number of researchers have applied multilevel decision-making techniques to handle product design, raw materials supply and inventory control problems in SCM. Yang, et al. [167] formulated a mixed 0-1 nonlinear bi-level decision model for the joint optimization of product family configuration and scaling design, in which a bi-level decision structure reveals coupled decision making between module configuration and parameter scaling. Based on a conditional value-at-risk $(\mathrm{CVaR})$ measure of risk management, $\mathrm{Xu}$, et al. [166] proposed a tri-level decision model for the three-echelon SCM in which the material supplier and the manufacturer maximize their own profit while the retailer maximizes its CVaR of the expected profit. The authors showed that the proposed 
tri-level decision model can be transformed into a bi-level decision model that can be solved by existing methods. Han, et al. [75] applied a linear TLMF decision model to describe a decentralized inventory control problem of a three-echelon hierarchical supply chain network composed of a sales company, two logistics centers and two manufacturing factories attached to each logistics center. They developed a TLMF Kth-Best algorithm to find an optimal solution to the resulting model.

\subsection{Traffic and transportation network design}

Severe traffic and transportation delays are incurred in most road networks as a result of continuously growing travel demand, increasing traffic congestion, transportation allocation problems between supply and demand nodes, and optimal transportation route problems. The rapid growth of overload in traffic and transportation networks has motivated decision makers to apply multilevel decision-making techniques to cope with the related decision-making and optimization problems in decentralized situations.

Extensive research on the basis of multilevel decision-making has been devoted to road network design problems as a result of insufficient provision of link capacity for travel demand surges. Cao, et al. [36] used a discrete bi-level decision model to describe the relationship of the benefit-cost of the traffic flow guidance system (TFGS) and the equilibrium of users, and presented an arithmetic based on sensitivity analysis. In a system which allows buses of different sizes to be assigned to public transport routes, dell'Olio, et al. [43] addressed a discrete bi-level optimization model with constraints on bus capacity to size buses and set frequencies on each route in an attempt to optimize the headways on each route in accordance with observed levels of demand. Ukkusuri, et al. [153] formulated a combined dynamic user equilibrium and traffic signal control problem as a discrete bi-level optimization model and solved the problem using a solution technique based on the iterative optimization and assignment method. Wang, et al. [158] addressed a discrete network design problem with multiple capacity levels which determines the optimal number of lanes to add to each candidate link in a road network. They formulated the problem as a bi-level decision model, where the upper level aims to minimize the total travel time by adding new lanes to candidate links and the lower level is a traditional Wardrop user equilibrium (UE) problem. Han, et al. [79] proposed a nonlinear bi-level decision model for traffic network signal control, which was formulated as a dynamic Stackelberg game and solved as a mathematical program with equilibrium constraints. Angulo, et al. [7] proposed a nonlinear bi-level decision model for the expansion of a 
highway network by adding several highway corridors within a geographical region, in which the upper level problem determines the location of the highway corridors by taking into account budgetary and technological restrictions, while the lower level problem models user behavior in the located transport network (choice of route and transport system). Fontaine and Minner [59] designed a linear bi-level decision model for the discrete network design problem which adds arcs to an existing road network at the leader stage and anticipates traffic equilibrium for the follower stage. They proposed a new fast solution method for the resulting model with binary leader and continuous follower variables under the assumption of partial cooperation.

In regard to solving transportation planning and scheduling, origin-destination allocation and routing optimization problems, Chiou [37] proposed a nonlinear bi-level decision model for a logistics network design problem with system-optimized flows and developed a novel solution algorithm to efficiently solve the problem. Ge, et al. [69] established a discrete bi-level decision model to analyze an integrated inventory-transportation optimization problem and adopted a layer-iterative algorithm to solve the resulting model. Liu, et al. [99] presented a novel real-time path planning approach for unmanned aerial vehicles, in which the planning problem is described as a nonlinear bi-level decision model. In particular, a discretization solution algorithm embedded with five heuristic optimization strategies was designed to speed up the planning. Konur and Golias [92] studied the scheduling of inbound trucks at the inbound doors of a cross-dock facility under truck arrival time uncertainty and formulated this problem as a pessimistic and optimistic discrete bi-level decision problem respectively. They developed a genetic algorithm to solve the bi-level formulations of the pessimistic and the optimistic approaches. Hajibabai, et al. [73] studied an integrated facility location problem that simultaneously considers traffic routing under congestion and pavement rehabilitation under deterioration and formulated this problem as a nonlinear mixed-integer bi-level program with facility location, freight shipment routing and pavement rehabilitation decisions in the upper level and traffic equilibrium in the lower level.

Researchers have also applied multilevel decision-making techniques to handle traffic and transportation problems under uncertainty. For example, Chiou [38] developed a bi-level decision support system for a normative road network design with uncertain travel demand in order to simultaneously reduce travel delay to road users and mitigate the vulnerability of the road network. $\mathrm{Xu}$ and Gang [164] investigated a transportation scheduling problem in a large-scale construction project under a fuzzy random environment and formulated this problem as a fuzzy and random 
bi-level multi-objective optimization model which was solved by a PSO algorithm. Shao, et al. [133] proposed a nonlinear bi-level optimization model to estimate the variation in peak hour origin-destination traffic demand from day-to-day hourly traffic counts throughout the whole year. A heuristic iterative estimation-assignment algorithm for solving the bi-level optimization problem was proposed.

\subsection{Energy management}

Growing environmental concerns have motivated worldwide attention to energy management. Multilevel decision-making techniques have been applied to handle many energy management problems, such as energy transmission and marketing, reducing pollution and promoting cleaner production.

In relation to the transmission and marketing of natural gas, Dempe, et al. [47] presented a mathematical framework for the problem of minimizing the cash-out penalties of a natural gas shipper and modeled the problem as a mixed-integer bi-level decision problem having one Boolean variable in the lower level problem, in which the decision making process for the shipper (leader) is to determine how to carry out its daily imbalances to minimize the penalty that will be imposed by the pipeline (follower). For the sake of justifying the daily imbalance swings made by the gas shipper as result of variations in the selling price of gas, Kalashnikov, et al. [88] extended the model presented by Dempe, et al. [47] to another bi-level optimization model, in which the upper level objective function includes additional terms that account for the gas shipping company's daily actions with the aim of taking advantage of the price variations. Dempe, et al. [48] also adopted a linear bi-level decision model to describe a natural gas cash-out problem between a natural gas shipping company and a pipeline operator and a penalty function method was developed to solve the model.

To handle marketing problems in electricity markets, Zhang, et al. [177] built a nonlinear bi-level optimization model for a strategic bidding problem in competitive day-ahead electricity markets and developed a PSO algorithm for solving the resulting model. Also, Zhang, et al. [178] presented a general nonlinear bi-level multi-leader one-follower decision model for strategic bidding optimization in day-ahead electricity markets. The resulting model allows each generating company to choose its biddings to maximize its individual profit; while a market operator can find its minimized purchase electricity fare, which is determined by the output power of each unit and 
the uniform marginal prices. The authors then developed a PSO algorithm to solve the problem. Garcés, et al. [68] presented a bi-level multi-follower decision model for electricity transmission expansion planning within a market environment. The upper-level problem represents the decisions to be made by the transmission planner with the target of deciding transmission investments while maximizing average social welfare and minimizing investment cost. The lower-level problems represent a market clearing for each market scenario and consider known investment decisions. Using duality theory, the proposed bi-level model was recast as a mixed-integer linear programming problem, which was solvable by branch-and-cut solvers. Fernandez-Blanco, et al. [58] discussed an alternative day-ahead auction based on consumer payment minimization for pool-based electricity markets and solved this problem by discrete bi-level optimization. In the upper-level optimization, generation is scheduled with the goal of minimizing the total consumer payment while taking into account the fact that locational marginal prices are determined by a multiperiod optimal power flow in the lower level. Hesamzadeh and Yazdani [83] proposed a mixed-integer linear bi-level multi-follower decision model for transmission planning in an environment where there is imperfect competition in the electricity supply industry, and the problem was solved using Kuhn-Tucker optimality conditions and a binary mapping approach. Street, et al. [148] developed a tri-level decision model for energy reserve scheduling in electricity markets with transmission flow limits and found a solution using a Benders decomposition approach. Fernandez-Blanco, et al. [57] presented a nonlinear mixed-integer bi-level decision-making formulation for alternative market-clearing procedures in restructured power systems that are dependent on market-clearing prices rather than on offers. Taha, et al. [149] presented a nonlinear bi-level optimization formulation for Quasi-Feed-In-Tariff (QFIT) policy which integrates the physical characteristics of the power-grid, in which the upper-level problem corresponds to the policy makers, whereas the lower-level decisions are made by generation companies.

Multilevel decision-making techniques have been also applied to handle water exchange problems in relation to the consumption of water resources and the generation of waste. Aviso, et al. [13] developed a fuzzy bi-level optimization model to explore the effect of charging fees for the purchase of freshwater and the treatment of wastewater in optimizing the water exchange network of plants in an eco-industrial park (EIP). Tan, et al. [150] extended the optimization model developed by Aviso, et al. [13] to a fuzzy bi-level decision model by modifying the role of the EIP authority to include water regeneration and redistribution via a centralized hub and found a 
reasonable compromise between the EIP authority's desire to minimize fresh water usage, and the participating companies' desire to minimize costs. Skulovich, et al. [146] presented a discrete bi-level optimization approach for the placement and sizing of closed surge tanks in the water distribution system subjected to transient events. Based on the optimization of comprehensive social, economic, agricultural, environment and groundwater preservation benefits, Guo, et al. [72] presented a bi-level multi-objective optimization model that allocates water resources rationally between all sectors and prevents over-exploitation.

\subsection{Safety and accident management}

Safety and accident management has increasingly attracted concern in relation to man-made disasters such as terrorist attacks and hazmat leakage, and natural disasters such as hurricanes and earthquakes. Multilevel decision-making techniques have been widely applied to assist authorities in making decisions associated with safety and accident management, e.g. electric power network defense, hazmat transportation, pollution abatement and emergency evacuation.

From the aspect of the prevention and defense of man-made and natural disaster, Yao, et al. [168] built a tri-level optimization model for resource allocation in electric power network defense which identifies the most critical network components to defend against possible terrorist attacks, and a decomposition approach was proposed to find an optimal solution to the resulting model. Alguacil, et al. [2] applied a tri-level decision model to describe an electric grid defense planning problem and solved it using a novel two-stage solution approach. Erkut and Gzara [54] proposed a discrete bi-level decision model for the problem of network design for hazardous material transportation where the government designates a network and the carriers choose the routes on the network. The authors developed a heuristic solution method that always finds a stable solution. Bianco, et al. [24] proposed a linear bi-level decision model for a hazmat transportation network design problem which was then transformed into a single-level mixed integer linear program by Kuhn-Tucker conditions for finding an optimal solution. Scaparra and Church [132] developed a mixed-integer bi-level program for critical infrastructure protection planning in which the upper-level problem involves the decisions about which facilities to fortify to minimize the worst-case efficiency reduction due to the loss of unprotected facilities, whereas worst-case scenario losses are modeled in the lower-level interdiction problem. He, et al. [80] presented two mixed integer bi-level decision-making models for integrated municipal solid waste management 
and greenhouse gas emissions control. Shih, et al. [140] applied nonlinear bi-level programming to determine a subsidy rate for Taiwan's domestic glass recycling industry. Hajinassiry, et al. [74] presented a new adaptive discrete bi-level optimization approach to solve a short-term hydrothermal coordination problem with AC (alternating current) network constraints.

To achieve emergency evacuation and provide rapid aid after a catastrophic disaster, Lv, et al. [105] proposed a bi-level optimization model to reduce traffic congestion of the transportation network while evacuating people to safe shelters during disasters or special events, in which the upper level aims to minimize the total evacuation time, while the lower level functions on the basis of user equilibrium assignment. A solution method based on discrete PSO and the Frank-Wolfe algorithm was employed to solve the bi-level optimization problem. Camacho-Vallejo, et al. [34] proposed a linear bi-level decision model for humanitarian logistics to optimize decisions related to the distribution of international aid after a catastrophic disaster. Apivatanagul, et al. [9] introduced nonlinear bi-level optimization for risk-based regional hurricane evacuation planning where the upper level develops an evacuation plan to minimize both risk and travel time while the lower level is a dynamic user equilibrium traffic assignment model. Ren, et al. [122] proposed a bi-level bi-objective decision model based on the concept of robust optimization for determining flows on emergency evacuation routes and traffic signals at intersections in the presence of uncertain background travel demands. A non-dominated sorting genetic algorithm was employed to determine the Pareto solutions of this optimization problem.

\section{Comprehensive analysis and prospective research topics}

In this survey, we have reviewed 179 papers and reported the new developments in multilevel decision-making techniques involving bi-level decision-making, tri-level decision-making, fuzzy multilevel decision-making, and their applications in handling real-world cases. These papers demonstrate the following features.

First, a large number of solution approaches involving exact algorithms and heuristic algorithms have been developed to solve different categories of multilevel decision problems. Detailed comparisons for the existing algorithms in relation to each category of multilevel decision problems are reported in Tables 1-7, which present advantages and disadvantages of the existing algorithms. It can be found that intelligence-based heuristic algorithms have been extensively used to solve various multilevel decision-making formulations in recent years especially for large-scale problems. 
In addition, researchers have proposed several new methods of constructing the set of usable multilevel decision-making test problems, which can be employed to illustrate the effectiveness of the proposed heuristic algorithms.

Second, motivated by the constant complexity of decision-making situations, an increasing number of papers are devoted to advanced multilevel decision-making techniques that involve bi-level decision-making with multiple optima, tri-level decision-making, and fuzzy multilevel decision-making. A range of decision models and effective solution algorithms have been developed to handle these complex situations.

Third, multilevel decision-making techniques have been widely applied to handle real-world problems. The application domains and multilevel decision-making techniques used in applications are summarized in Table 8, which shows the number of each category of multilevel decision-making techniques applied in each domain. Although the majority of these application papers use basic bi-level decision-making techniques, advanced multilevel decision-making techniques have been increasingly applied in recent years since many real-world cases occur in highly complex decision situations, e.g. where multiple followers are involved and there is uncertainty in data. Moreover, the number of papers in respect of applications (47 papers) has experienced a faster upward trend than the number of theoretical research papers (36 papers) since 2010.

Although multilevel decision-making techniques have been the subject of great developments, several challenges still require further research.

First, computational complexity is a crucial issue in solving multilevel decision problems. Although many solution algorithms have been developed, it is necessary and urgent to develop much more efficient algorithms for solving advanced multilevel decision problems, in particular in large-scale versions. Moreover, many multilevel decision problems in applications may have no optimal solutions based on existing solution concepts. How to find an usable or satisfactory solution to those real-world multilevel decision problems is also an emerging research topic with respect to computational complexity.

Second, multilevel decision problems nowadays often appear in highly complex and diversified decision environments where decision makers sometimes need to make an optimal or a wise decision from big data with uncertainty; thus, advanced multilevel decision-making techniques need to be explored in depth. This also requires further research on how to wisely model such problems 
and implement data-driven decision-making in the current age of big data by means of a multilevel decision support system.

Lastly, many multilevel decision problems in the real world cannot be modeled as mathematical programming formulations. Consequently, it is another challenge to break through mathematical programs and integrate the principle of multilevel decision-making with other decision-making techniques to handle a much wider range of decision problems.

Two important features of the paper clearly distinguish it from other survey papers in the area of multilevel decision-making. On the one hand, in contrast to other survey papers that only review early research on basic bi-level programming, this survey targets the latest multilevel decision-making techniques and their comprehensive development, in particular new developments in bi-level decision-making with multiple optima, tri-level decision-making, and fuzzy multilevel decision-making. On the other hand, it systematically examines the real-world application developments of multilevel decision-making techniques and clusters the applications into four major domains. We believe that this paper provides researchers and practitioners with state-of-the-art knowledge on the development of multilevel decision-making techniques and gives guidelines about how to apply multilevel decision-making techniques to deal with various decision activities in different domains.

\section{Acknowledgements}

This work is supported by the Australian Research Council (ARC) under discovery grant DP140101366, and the National High Technology Research and Development Program of China (NO. 2013AA040402). 
Table 1 Summary of algorithms developed for solving linear bi-level decision problems

\begin{tabular}{|c|c|c|c|c|c|}
\hline Algorithm Type & Algorithm name & Reference & Period & Advantage & Disadvantage \\
\hline \multirow[t]{5}{*}{ Exact algorithm } & Vertex enumeration & {$[23,35,135,152]$} & $1982-2005$ & Can find an optimal solution if it exists. & Time-consuming for solving large-scale problems. \\
\hline & Branch-and-bound & {$[18,19,60,136]$} & $1981-2006$ & & \\
\hline & Complementary pivot & {$[23,85,115]$} & 1984-1993 & & \\
\hline & Penalty function & {$[6,163]$} & 1990-1993 & & \\
\hline & Disjunctive cuts & [11] & 2007 & & \\
\hline Exact algorithm & Branch-and-cut & [12] & 2007 & $\begin{array}{l}\text { Outperforms the branch-and-bound algorithm in } \\
\text { terms of computing times. }\end{array}$ & Time-consuming for solving large-scale problems. \\
\hline Exact algorithm & Simplex pivots & [71] & 2009 & $\begin{array}{l}\text { More effective than the branch-and-bound algorithm } \\
\text { when the number of leader variables is small. }\end{array}$ & Time-consuming for solving large-scale problems. \\
\hline \multirow[t]{2}{*}{ Exact algorithm } & Ranking extreme points & {$[30]$} & 2012 & Can solve problems with interval coefficients in & Time-consuming for solving large-scale problems. \\
\hline & Cutting plane & [121] & 2014 & objective functions. & \\
\hline \multirow[t]{2}{*}{ Heuristic algorithm } & Tabu search & {$[70]$} & 1996 & Is applied to solve large-scale problems. & May be convergent to a local solution; \\
\hline & Genetic algorithm & {$[33,82]$} & 2002,2008 & & $\begin{array}{l}\text { Are limited to solving linear problems that all } \\
\text { decision entities share the same constraint conditions. }\end{array}$ \\
\hline \multirow[t]{2}{*}{ Heuristic algorithm } & Neural network and Tabu search & [96] & 2007 & Can find a solution. & May be convergent to a local solution; \\
\hline & PSO & [94] & 2009 & & $\begin{array}{l}\text { Are limited to solving linear problems that all } \\
\text { decision entities share the same constraint conditions } \\
\text { Are not applied to solve large-scale problems. }\end{array}$ \\
\hline Heuristic algorithm & Neural network & [84] & 2010 & Can find a solution. & $\begin{array}{l}\text { May be convergent to a local solution; } \\
\text { Is not applied to solve large-scale problems. }\end{array}$ \\
\hline
\end{tabular}


Table 2 Summary of algorithms developed for solving nonlinear bi-level decision problems

\begin{tabular}{|c|c|c|c|c|c|}
\hline Algorithm type & Algorithm name & Reference & Period & Advantage & Disadvantage \\
\hline \multirow[t]{4}{*}{ Exact algorithm } & Branch-and-bound & {$[1,14,51]$} & $1988-1992$ & Can find an optimal solution if it exists. & Time-consuming for solving large-scale problems; \\
\hline & Decent method & [154] & 1994 & & Cannot solve problems in which the lower-level \\
\hline & Monotonic optimization & [151] & 2007 & & problem is nonconvex programming. \\
\hline & Direct search & [109] & 2011 & & \\
\hline Exact algorithm & Bounding algorithm & [111] & 2008 & $\begin{array}{l}\text { Can solve problems involving nonconvex objective } \\
\text { functions in both decision levels. }\end{array}$ & Time-consuming for solving large-scale problems. \\
\hline \multirow[t]{3}{*}{ Heuristic algorithm } & Evolutionary algorithm & [159] & 2005 & Can find a solution. & May be convergent to a local solution; \\
\hline & PSO and chaos search & [157] & 2013 & & Is not applied to solve large-scale problems; \\
\hline & Neural network & {$[81,106,107]$} & $2008-2014$ & & $\begin{array}{l}\text { Cannot solve problems in which the lower-level } \\
\text { problem is nonconvex programming. }\end{array}$ \\
\hline Heuristic algorithm & Estimation of distribution algorithm & [156] & 2014 & $\begin{array}{l}\text { Can find a solution; } \\
\text { Is applied to solve large-scale problems. }\end{array}$ & $\begin{array}{l}\text { May be convergent to a local solution; } \\
\text { Cannot solve problems in which the lower-level } \\
\text { problem is nonconvex programming. }\end{array}$ \\
\hline
\end{tabular}


Table 3 Summary of algorithms developed for solving discrete bi-level decision problems

\begin{tabular}{|c|c|c|c|c|c|}
\hline Algorithm type & Algorithm name & Reference & Period & Advantage & Disadvantage \\
\hline \multirow[t]{3}{*}{ Exact algorithm } & Branch-and-bound & {$[20,112,161]$} & $1990-1992$ & \multirow[t]{3}{*}{ Can solve discrete linear problems. } & Time-consuming for solving large-scale problems; \\
\hline & Penalty function & {$[155]$} & 1996 & & Cannot solve discrete nonlinear problems. \\
\hline & Parametric programming & {$[55,87]$} & 2007,2010 & & \\
\hline \multirow[t]{2}{*}{ Exact algorithm } & \multirow[t]{2}{*}{ Branch-and-bound } & \multirow[t]{2}{*}[50]{} & \multirow[t]{2}{*}{1992} & Can solve discrete nonlinear problems involving & Time-consuming for solving large-scale problems. \\
\hline & & & & convex objective functions in both decision levels. & \\
\hline \multirow[t]{2}{*}{ Exact algorithm } & Multiparametric programming & [49] & 2010 & \multirow[t]{2}{*}{ Can solve discrete nonlinear problems. } & \multirow[t]{2}{*}{ Time-consuming for solving large-scale problems. } \\
\hline & Bounding algorithm & [110] & 2010 & & \\
\hline \multirow[t]{2}{*}{ Exact algorithm } & \multirow[t]{2}{*}{ The algorithm $\mathrm{Alg}^{\mathrm{BMILP}}$} & \multirow[t]{2}{*}{ [165] } & \multirow[t]{2}{*}{2014} & Can solve discrete linear problems; & \multirow[t]{2}{*}{ Cannot solve discrete nonlinear problems. } \\
\hline & & & & Is applied to solve large-scale problems. & \\
\hline \multirow[t]{2}{*}{ Exact algorithm } & \multirow[t]{2}{*}{ Iterative algorithm } & \multirow[t]{2}{*}[134]{} & \multirow[t]{2}{*}{2014} & Can solve discrete linear problems in which the & Cannot solve discrete nonlinear problems; \\
\hline & & & & objective function of the first level is linear fractional. & Time-consuming for solving large-scale problems. \\
\hline \multirow[t]{3}{*}{ Heuristic algorithm } & \multirow[t]{3}{*}{ Simple tabu search } & \multirow[t]{3}{*}{ [160] } & \multirow[t]{3}{*}{1996} & \multirow[t]{3}{*}{ Can find a solution to discrete linear problems. } & May be convergent to a local solution; \\
\hline & & & & & Is not applied to solve discrete nonlinear and \\
\hline & & & & & large-scale problems. \\
\hline \multirow[t]{2}{*}{ Heuristic algorithm } & \multirow[t]{2}{*}{ Genetic algorithm } & \multirow[t]{2}{*}{ [114] } & \multirow[t]{2}{*}{2005} & Can find a solution to discrete linear problems; & May be convergent to a local solution; \\
\hline & & & & Is applied to solve large-scale problems. & Is not applied to solve discrete nonlinear problems. \\
\hline
\end{tabular}


Table 4 Summary of algorithms developed for solving bi-level multi-objective decision problems

\begin{tabular}{|c|c|c|c|c|c|}
\hline Algorithm type & Algorithm name & Reference & Period & Advantage & Disadvantage \\
\hline Exact algorithm & Exact penalty method & [8] & 2009 & $\begin{array}{l}\text { Can solve linear bi-level decision problems where the } \\
\text { lower level is a multi-objective optimization problem. }\end{array}$ & Is not applied to solve nonlinear and large-scale problems. \\
\hline Exact algorithm & $\begin{array}{l}\text { Weighted sum } \\
\text { scalarization methods and } \\
\text { scalarization methods }\end{array}$ & [28] & 2010 & $\begin{array}{l}\text { Can solve linear bi-level decision problems where the } \\
\text { upper level is a multi-objective optimization problem. }\end{array}$ & Is not applied to solve nonlinear and large-scale problems. \\
\hline Exact algorithm & Interactive approach & [53] & 2013 & Can solve integer fractional BLMO problems. & Is not applied to solve nonlinear and large-scale problems. \\
\hline Exact algorithm & $\begin{array}{l}\text { Iterative method using } \\
\text { sensitivity theorems }\end{array}$ & [52] & 2010 & Can solve nonlinear nonconvex BLMO problems & Is not applied to solve nonlinear and large-scale problems. \\
\hline Heuristic algorithm & PSO & [3] & 2014 & $\begin{array}{l}\text { Can solve linear bi-level decision problems where the } \\
\text { upper level is a multi-objective optimization problem; } \\
\text { Is applied to solve large-scale problems. }\end{array}$ & May be convergent to a local solution. \\
\hline Heuristic algorithm & Genetic algorithm & [29] & 2011 & $\begin{array}{l}\text { Can solve linear bi-level decision problems where the } \\
\text { lower level is a multi-objective optimization problem; } \\
\text { Is applied to solve large-scale problems. }\end{array}$ & May be convergent to a local solution. \\
\hline Heuristic algorithm & $\begin{array}{l}\text { PSO } \\
\text { Evolutionary-cum-local-search }\end{array}$ & $\begin{array}{l}{[179]} \\
{[42]}\end{array}$ & $\begin{array}{l}2013 \\
2010\end{array}$ & $\begin{array}{l}\text { Can solve nonlinear BLMO decision problems; } \\
\text { Is applied to solve large-scale problems. }\end{array}$ & May be convergent to a local solution. \\
\hline
\end{tabular}


Table 5 Summary of algorithms developed for solving bi-level multi-leader and/or multi-follower decision problems

\begin{tabular}{|c|c|c|c|c|c|}
\hline Algorithm type & Algorithm name & Reference & Period & Advantage & Disadvantage \\
\hline \multirow[t]{3}{*}{ Exact algorithm } & $K$ th-Best & {$[137,138]$} & 2005,2007 & \multirow{3}{*}{$\begin{array}{l}\text { Can solve linear BLMF decision problems with different } \\
\text { relationships between multiple followers. }\end{array}$} & \multirow{3}{*}{$\begin{array}{l}\text { Is not applied to solve nonlinear and large-scale } \\
\text { problems. }\end{array}$} \\
\hline & Kuhn-Tucker & [102] & 2007 & & \\
\hline & Branch-and-bound & {$[100]$} & 2007 & & \\
\hline \multirow[t]{5}{*}{ Heuristic algorithm } & \multirow[t]{3}{*}{$\mathrm{PSO}$} & \multirow[t]{3}{*}[63]{} & \multirow[t]{3}{*}{2010} & Can solve linear and nonlinear BLML, BLMF and & May be convergent to a local solution; \\
\hline & & & & BLMLMF decision problems; & Is not applied to solve large-scale problems. \\
\hline & & & & Is implemented into a decision support system (software). & \\
\hline & \multirow[t]{2}{*}{ Evolutionary algorithm } & \multirow[t]{2}{*}{ [142] } & \multirow[t]{2}{*}{2014} & $\begin{array}{l}\text { Can solve BLMLMF decision problems with nonlinear and } \\
\text { discrete variables; }\end{array}$ & \multirow[t]{2}{*}{ May be convergent to a local solution. } \\
\hline & & & & Is applied to solve large-scale problems. & \\
\hline
\end{tabular}

Table 6 Summary of algorithms developed for solving tri-level decision problems

\begin{tabular}{|c|c|c|c|c|c|}
\hline Algorithm type & Algorithm name & Reference & Period & Advantage & Disadvantage \\
\hline \multirow[t]{4}{*}{ Exact algorithm } & Cutting plane & [15] & 1984 & \multirow[t]{4}{*}{ Can find a solution to linear problems. } & \multirow{4}{*}{$\begin{array}{l}\text { May find a local solution rather than an optimal } \\
\text { solution; }\end{array}$} \\
\hline & Penalty function & {$[162]$} & 1997 & & \\
\hline & Kuhn-Tucker & {$[4,143]$} & 1988,2001 & & \\
\hline & Multiparametric programming & {$[56]$} & 2007 & & \\
\hline Exact algorithm & $K$ th-Best & {$[176]$} & 2010 & $\begin{array}{l}\text { Can find an optimal solution to linear problems; } \\
\text { Is implemented into a decision support system } \\
\text { (software). }\end{array}$ & $\begin{array}{l}\text { Cannot solve nonlinear problems; } \\
\text { Is not applied to solve large-scale problems. }\end{array}$ \\
\hline Fuzzy approach & $\begin{array}{l}\text { Interactive fuzzy programming } \\
\text { Fuzzy goal programming }\end{array}$ & $\begin{array}{l}{[95,128,139,144,145]} \\
{[10,119]}\end{array}$ & $\begin{array}{l}1996-2003 \\
2007,2009\end{array}$ & Can find a solution to linear problems. & $\begin{array}{l}\text { Are limited to solve problems where all decision } \\
\text { entities prefer to cooperate with one another; } \\
\text { May find a satisfactory solution rather than an } \\
\text { optimal solution; } \\
\text { Are not applied to solve large-scale problems. }\end{array}$ \\
\hline Heuristic algorithm & PSO & [77] & 2015 & $\begin{array}{l}\text { Can find a solution to linear and nonlinear problems; } \\
\text { Can find better solutions than fuzzy approaches. }\end{array}$ & $\begin{array}{l}\text { May be convergent to a local solution; } \\
\text { Is not applied to solve large-scale problems. }\end{array}$ \\
\hline
\end{tabular}


Table 7 Summary of algorithms developed for solving fuzzy multilevel decision problems

\begin{tabular}{|c|c|c|c|c|c|}
\hline Algorithm type & Algorithm name & Reference & Period & Advantage & Disadvantage \\
\hline \multirow[t]{4}{*}{$\begin{array}{l}\text { Approximation } \\
\text { approach }\end{array}$} & Approximation Kuhn-Tucker & [169] & 2005 & Can solve fuzzy linear bi-level decision problems. & $\begin{array}{l}\text { Are limited to handling special membership } \\
\text { functions; }\end{array}$ \\
\hline & Approximation $K$ th-Best & {$[26,170,171]$} & $2007-2013$ & $\begin{array}{l}\text { Can solve fuzzy linear bi-level decision problems } \\
\text { and fuzzy linear BLMOMF decision problems. }\end{array}$ & $\begin{array}{l}\text { Are not applied to solve nonlinear and } \\
\text { large-scale problems. }\end{array}$ \\
\hline & Approximation branch-and-bound & {$[172-174]$} & $2007-2008$ & $\begin{array}{l}\text { Can solve fuzzy linear BLMO decision problems } \\
\text { and fuzzy linear BLMOMF decision problems. }\end{array}$ & \\
\hline & Programmable $\lambda$-cut approximation & {$[65,67]$} & 2008,2010 & $\begin{array}{l}\text { Can solve fuzzy linear bi-level decision problems } \\
\text { and fuzzy linear BLMO decision problems; } \\
\text { Is implemented into a decision support system } \\
\text { (software). }\end{array}$ & \\
\hline \multirow[t]{2}{*}{ Fuzzy approach } & Interactive fuzzy programming & $\begin{array}{l}{[116,125,} \\
127,129,130]\end{array}$ & $2000-2004$ & $\begin{array}{l}\text { Can solve fuzzy linear bi-level decision problems, } \\
\text { fuzzy linear multilevel fractional decision } \\
\text { problems, fuzzy multilevel 0-1 decision problems, } \\
\text { fuzzy multilevel nonconvex decision problems and } \\
\text { fuzzy nonlinear tri-level decision problems with } \\
\text { multiple objectives. }\end{array}$ & $\begin{array}{l}\text { Are limited to solve problems where all decision } \\
\text { entities prefer to cooperate with one another; } \\
\text { May find a satisfactory solution rather than an } \\
\text { optimal solution; } \\
\text { Are not applied to solve large-scale problems. }\end{array}$ \\
\hline & Fuzzy goal programming & [118] & 2014 & Can solve fuzzy linear bi-level decision problems. & \\
\hline Heuristic algorithm & PSO & {$[64]$} & 2009 & Can solve fuzzy linear BLMF decision problems. & $\begin{array}{l}\text { May be convergent to a local solution; } \\
\text { Is not applied to solve nonlinear and large-scale } \\
\text { problems. }\end{array}$ \\
\hline Heuristic algorithm & Neural network and genetic algorithm & {$[62]$} & 2005 & $\begin{array}{l}\text { Can solve fuzzy nonlinear BLMF decision } \\
\text { problems. }\end{array}$ & $\begin{array}{l}\text { May be convergent to a local solution; } \\
\text { Is not applied to solve large-scale problems. }\end{array}$ \\
\hline
\end{tabular}


Table 8 Summary of multilevel decision-making techniques in each application domain

\begin{tabular}{|c|c|c|c|c|c|c|c|c|}
\hline \multirow[b]{2}{*}{ Application domains } & \multicolumn{8}{|c|}{ Decision-making techniques } \\
\hline & Linear bi-level & Nonlinear bi-level & Discrete bi-level & BLMO & BLML/BLMF & Tri-level & Fuzzy multilevel & No. of listed references \\
\hline Supply chain management & 1 & 3 & 8 & 2 & & 2 & & 16 \\
\hline Traffic and transportation network design & 1 & 5 & 7 & & & & 2 & 15 \\
\hline Energy management & 1 & 2 & 6 & 1 & 2 & 1 & 2 & 15 \\
\hline Safety and accident management & 2 & 2 & 5 & 1 & & 2 & & 12 \\
\hline Total & 5 & 12 & 26 & 4 & 2 & 5 & 4 & 58 \\
\hline
\end{tabular}




\section{References}

[1] F. Al-Khayyal, R. Horst, P. Pardalos, Global optimization of concave functions subject to quadratic constraints: An application in nonlinear bilevel programming, Annals of Operations Research, 34 (1992) 125-147.

[2] N. Alguacil, A. Delgadillo, J.M. Arroyo, A trilevel programming approach for electric grid defense planning, Computers \& Operations Research, 41 (2014) 282-290.

[3] M.J. Alves, J.P. Costa, An algorithm based on particle swarm optimization for multiobjective bilevel linear problems, Applied Mathematics and Computation, 247 (2014) 547-561.

[4] G. Anandalingam, A mathematical programming model of decentralized multi-level systems, Journal of the Operational Research Society, 39 (1988) 1021-1033.

[5] G. Anandalingam, V. Apprey, Multi-level programming and conflict resolution, European Journal of Operational Research, 51 (1991) 233-247.

[6] G. Anandalingam, D.J. White, A solution method for the linear static Stackelberg problem using penalty functions, IEEE Transactions on Automatic Control, 35 (1990) 1170-1173.

[7] E. Angulo, E. Castillo, R. García-Ródenas, J. Sánchez-Vizcaíno, A continuous bi-level model for the expansion of highway networks, Computers \& Operations Research, 41 (2014) 262-276.

[8] Z. Ankhili, A. Mansouri, An exact penalty on bilevel programs with linear vector optimization lower level, European Journal of Operational Research, 197 (2009) 36-41.

[9] P. Apivatanagul, R. Davidson, L. Nozick, Bi-level optimization for risk-based regional hurricane evacuation planning, Natural Hazards, 60 (2012) 567-588.

[10] S.R. Arora, R. Gupta, Interactive fuzzy goal programming approach for bilevel programming problem, European Journal of Operational Research, 194 (2009) 368-376.

[11] C. Audet, J. Haddad, G. Savard, Disjunctive cuts for continuous linear bilevel programming, Optimization Letters, 1 (2007) 259-267.

[12] C. Audet, G. Savard, W. Zghal, New Branch-and-Cut Algorithm for Bilevel Linear Programming, Journal of Optimization Theory and Applications, 134 (2007) 353-370.

[13] K.B. Aviso, R.R. Tan, A.B. Culaba, J.B. Cruz Jr, Bi-level fuzzy optimization approach for water exchange in eco-industrial parks, Process Safety \& Environmental Protection: Transactions of the Institution of Chemical Engineers Part B, 88 (2010) 31-40.

[14] J. Bard, Convex two-level optimization, Mathematical Programming, 40 (1988) 15-27.

[15] J.F. Bard, An investigation of the linear three level programming problem, IEEE Transactions on Systems, Man, and Cybernetics, SMC-14 (1984) 711-717.

[16] J.F. Bard, Some properties of the bilevel programming problem, Journal of Optimization Theory and Applications, 68 (1991) 371-378.

[17] J.F. Bard, Practical Bilevel Optimization: Algorithms and Applications, Kluwer Academic Publishers, Dordrecht, The Netherlands, 1998.

[18] J.F. Bard, J.E. Falk, An explicit solution to the multi-level programming problem, Computers \& Operations Research, 9 (1982) 77-100.

[19] J.F. Bard, J.T. Moore, A branch and bound algorithm for the bilevel programming problem, SIAM Journal on Scientific and Statistical Computing, 11 (1990) 281-292.

[20] J.F. Bard, J.T. Moore, An algorithm for the discrete bilevel programming problem, Naval Research Logistics (NRL), 39 (1992) 419-435.

[21] O. Ben-Aved, C.E. Blair, Computational difficulties of bilevel linear programming, Operations Research, 38 (1990) 556-560.

[22] O. Ben-Ayed, Bilevel linear programming, Computers \& Operations Research, 20 (1993) 485-501. 
[23] W. Bialas, M.H. Karwan, Two-level linear programming, Management Science, 30 (1984) 1004-1020.

[24] L. Bianco, M. Caramia, S. Giordani, A bilevel flow model for hazmat transportation network design, Transportation Research Part C: Emerging Technologies, 17 (2009) 175-196.

[25] J. Bracken, J.T. McGill, Mathematical programs with optimization problems in the constraints, Operations Research, 21 (1973) 37-44.

[26] A. Budnitzki, The solution approach to linear fuzzy bilevel optimization problems, Optimization, (2013) 1-15.

[27] H.I. Calvete, C. Galé, Linear bilevel multi-follower programming with independent followers, Journal of Global Optimization, 39 (2007) 409-417.

[28] H.I. Calvete, C. Galé, Linear bilevel programs with multiple objectives at the upper level, Journal of Computational and Applied Mathematics, 234 (2010) 950-959.

[29] H.I. Calvete, C. Galé, On linear bilevel problems with multiple objectives at the lower level, Omega, 39 (2011) $33-40$.

[30] H.I. Calvete, C. Galé, Linear bilevel programming with interval coefficients, Journal of Computational and Applied Mathematics, 236 (2012) 3751-3762.

[31] H.I. Calvete, C. Galé, J.A. Iranzo, Planning of a decentralized distribution network using bilevel optimization, Omega, 49 (2014) 30-41.

[32] H.I. Calvete, C. Galé, M.-J. Oliveros, Bilevel model for production-distribution planning solved by using ant colony optimization, Computers \& Operations Research, 38 (2011) 320-327.

[33] H.I. Calvete, C. Galee, P.M. Mateo, A new approach for solving linear bilevel problems using genetic algorithms, European Journal of Operational Research, 188 (2008) 14-28.

[34] J.-F. Camacho-Vallejo, E. González-Rodríguez, F.J. Almaguer, R.G. González-Ramírez, A bi-level optimization model for aid distribution after the occurrence of a disaster, Journal of Cleaner Production, 105 (2015) 134-145.

[35] W. Candler, R. Townsley, A linear two-level programming problem, Computers \& Operations Research, 9 (1982) 59-76.

[36] S. Cao, Z. Yuan, Y. Li, X. Wu, Model for road network stochastic user equilibrium based on bi-level programming under the action of the traffic flow guidance system, Journal of Transportation Systems Engineering and Information Technology, 7 (2007) 36-42.

[37] S.-W. Chiou, A bi-level programming for logistics network design with system-optimized flows, Information Sciences, 179 (2009) 2434-2441.

[38] S.-W. Chiou, A bi-level decision support system for uncertain network design with equilibrium flow, Decision Support Systems, 69 (2015) 50-58.

[39] B. Colson, P. Marcotte, G. Savard, Bilevel programming: A survey, 4OR, 3 (2005) 87-107.

[40] B. Colson, P. Marcotte, G. Savard, An overview of bilevel optimization, Annals of Operations Research, 153 (2007) 235-256.

[41] K. Deb, A. Sinha, Constructing test problems for bilevel evolutionary multi-objective optimization, in: IEEE Congress on Evolutionary Computation, 2009, pp. 1153-1160.

[42] K. Deb, A. Sinha, An efficient and accurate solution methodology for bilevel multi-objective programming problems using a hybrid evolutionary-local-search algorithm, Evolutionary Computation, 18 (2010) 403-449.

[43] L. dell’Olio, A. Ibeas, F. Ruisánchez, Optimizing bus-size and headway in transit networks, Transportation, 39 (2012) 449-464.

[44] V. DeMiguel, X. Huifu, A stochastic multiple-leader Stackelberg model: Analysis, computation, and application, Operations Research, 57 (2009) 1220-1235.

[45] S. Dempe, Foundations of Bilevel Programming, Kluwer Academic Publishers, Dordrecht, The Netherlands, 2002.

[46] S. Dempe, Comment to "interactive fuzzy goal programming approach for bilevel programming problem" by S.R. Arora and R. Gupta, European Journal of Operational Research, 212 (2011) 429-431.

[47] S. Dempe, V. Kalashnikov, R.Z. Ríos-Mercado, Discrete bilevel programming: Application to a natural gas cash-out 
problem, European Journal of Operational Research, 166 (2005) 469-488.

[48] S. Dempe, V.V. Kalashnikov, G.A. Pérez-Valdés, N.I. Kalashnykova, Natural gas bilevel cash-out problem: Convergence of a penalty function method, European Journal of Operational Research, 215 (2011) 532-538.

[49] L.F. Domínguez, E.N. Pistikopoulos, Multiparametric programming based algorithms for pure integer and mixed-integer bilevel programming problems, Computers \& Chemical Engineering, 34 (2010) 2097-2106.

[50] T. Edmunds, J. Bard, An algorithm for the mixed-integer nonlinear bilevel programming problem, Annals of Operations Research, 34 (1992) 149-162.

[51] T.A. Edmunds, J.F. Bard, Algorithms for nonlinear bilevel mathematical programs, IEEE Transactions on Systems, Man and Cybernetics, 21 (1991) 83-89.

[52] G. Eichfelder, Multiobjective bilevel optimization, Mathematical Programming, 123 (2010) 419-449.

[53] O.E. Emam, Interactive approach to bi-level integer multi-objective fractional programming problem, Applied Mathematics and Computation, 223 (2013) 17-24.

[54] E. Erkut, F. Gzara, Solving the hazmat transport network design problem, Computers \& Operations Research, 35 (2008) 2234-2247.

[55] N.P. Faísca, V. Dua, B. Rustem, P. Saraiva, E. Pistikopoulos, Parametric global optimisation for bilevel programming, Journal of Global Optimization, 38 (2007) 609-623.

[56] N.P. Faísca, P.M. Saraiva, B. Rustem, E.N. Pistikopoulos, A multi-parametric programming approach for multilevel hierarchical and decentralised optimisation problems, Computational Management Science, 6 (2007) 377-397.

[57] R. Fernandez-Blanco, J.M. Arroyo, N. Alguacil, A unified bilevel programming framework for price-based market clearing under marginal pricing, IEEE Transactions on Power Systems, 27 (2012) 517-525.

[58] R. Fernandez-Blanco, J.M. Arroyo, N. Alguacil, Network-constrained day-ahead auction for consumer payment minimization, IEEE Transactions on Power Systems, 29 (2014) 526-536.

[59] P. Fontaine, S. Minner, Benders decomposition for discrete-continuous linear bilevel problems with application to traffic network design, Transportation Research Part B: Methodological, 70 (2014) 163-172.

[60] J. Fortuny-Amat, B. McCarl, A representation and economic interpretation of a two-level programming problem, The Journal of the Operational Research Society, 32 (1981) 783-792.

[61] J. Gang, Y. Tu, B. Lev, J. Xu, W. Shen, L. Yao, A multi-objective bi-level location planning problem for stone industrial parks, Computers \& Operations Research, 56 (2015) 8-21.

[62] J. Gao, B. Liu, Fuzzy multilevel programming with a hybrid intelligent algorithm, Computers \& Mathematics with Applications, 49 (2005) 1539-1548.

[63] Y. Gao, Bi-level decision making with fuzzy sets and particle swarm optimisation, in: Faculty of Engineering and Information Technology, University of Technology Sydney, 2010.

[64] Y. Gao, G. Zhang, J. Lu, A particle swarm optimization based algorithm for fuzzy bilevel decision making with constraints-shared followers, in: The ACM symposium on Applied Computing, ACM, Honolulu, Hawaii, 2009, pp. 1075-1079.

[65] Y. Gao, G. Zhang, J. Lu, T. Dillon, X. Zeng, A $\lambda$-cut-approximate algorithm for goal-based bilevel risk management systems, International Journal of Information Technology and Decision Making, 7 (2008) 589-610.

[66] Y. Gao, G. Zhang, J. Lu, H.M. Wee, Particle swarm optimization for bi-level pricing problems in supply chains, Journal of Global Optimization, 51 (2011) 245-254.

[67] Y. Gao, G. Zhang, J. Ma, J. Lu, A $\lambda$-cut and goal programming based algorithm for fuzzy linear multiple objective bi-level optimization, IEEE Transactions on Fuzzy Systems, 18 (2010) 1-13.

[68] L.P. Garcés, A.J. Conejo, R. Garcia-Bertrand, R. Romero, A bilevel approach to transmission expansion planning within a market environment, IEEE Transactions on Power Systems, 24 (2009) 1513-1522.

[69] X. Ge, Y. Chen, W. Wang, Model and algorithm for inventory-transportation integrated optimization based on bi-level programming, International Journal of Advancements in Computing Technology, 5 (2013) 460-468.

[70] M. Gendreau, P. Marcotte, G. Savard, A hybrid Tabu-ascent algorithm for the linear bilevel programming problem, 
Journal of Global Optimization, 8 (1996) 217-233.

[71] J. Glackin, J.G. Ecker, M. Kupferschmid, Solving bilevel linear programs using multiple objective linear programming, Journal of Optimization Theory and Applications, 140 (2009) 197-212.

[72] Z. Guo, J. Chang, Q. Huang, L. Xu, C. Da, H. Wu, Bi-level optimization allocation model of water resources for different water industries, Water Science \& Technology: Water Supply, 14 (2014) 470-477.

[73] L. Hajibabai, Y. Bai, Y. Ouyang, Joint optimization of freight facility location and pavement infrastructure rehabilitation under network traffic equilibrium, Transportation Research Part B: Methodological, 63 (2014) 38-52.

[74] M. Hajinassiry, N. Amjady, H. Sharifzadeh, Hydrothermal coordination by bi-level optimization and composite constraint handling method, International Journal of Electrical Power \& Energy Systems, 62 (2014) 476-489.

[75] J. Han, J. Lu, Y. Hu, G. Zhang, Tri-level decision-making with multiple followers: Model, algorithm and case study, Information Sciences, 311 (2015) 182-204.

[76] J. Han, J. Lu, G. Zhang, S. Ma, Multi-follower tri-level decision making with uncooperative followers, in: The 11th International FLINS Conference, Brazil, 2014, pp. 524-529.

[77] J. Han, G. Zhang, Y. Hu, J. Lu, Solving tri-level programming problems using a particle swarm optimization algorithm, in: The 10th IEEE Conference on Industrial Electronics and Applications, 2015, pp. 569-574.

[78] J. Han, G. Zhang, J. Lu, Y. Hu, S. Ma, Model and algorithm for multi-follower tri-level hierarchical decision-making, in: Neural Information Processing, Springer International Publishing, 2014, pp. 398-406.

[79] K. Han, Y. Sun, H. Liu, T.L. Friesz, T. Yao, A bi-level model of dynamic traffic signal control with continuum approximation, Transportation Research Part C: Emerging Technologies, 55 (2015) 409-431.

[80] L. He, G.H. Huang, H. Lu, Greenhouse gas emissions control in integrated municipal solid waste management through mixed integer bilevel decision-making, Journal of Hazardous Materials, 193 (2011) 112-119.

[81] X. He, C. Li, T. Huang, C. Li, Neural network for solving convex quadratic bilevel programming problems, Neural Networks, 51 (2014) 17-25.

[82] S.R. Hejazi, A. Memariani, G. Jahanshahloo, M.M. Sepehri, Linear bilevel programming solution by genetic algorithm, Computers \& Operations Research, 29 (2002) 1913-1925.

[83] M.R. Hesamzadeh, M. Yazdani, Transmission capacity expansion in imperfectly competitive power markets, IEEE Transactions on Power Systems, 29 (2014) 62-71.

[84] T. Hu, X. Guo, X. Fu, Y. Lv, A neural network approach for solving linear bilevel programming problem, Knowledge-Based Systems, 23 (2010) 239-242.

[85] J.J. Júdice, A.M. Faustino, A sequential LCP method for bilevel linear programming, Annals of Operations Research, 34 (1992) 89-106.

[86] H. Küçükaydin, N. Aras, I. Kuban Altınel, Competitive facility location problem with attractiveness adjustment of the follower: A bilevel programming model and its solution, European Journal of Operational Research, 208 (2011) 206-220.

[87] M. Köppe, M. Queyranne, C.T. Ryan, Parametric integer programming algorithm for bilevel mixed integer programs, Journal of Optimization Theory and Applications, 146 (2010) 137-150.

[88] V. Kalashnikov, G. Pérez, N. Kalashnykova, A linearization approach to solve the natural gas cash-out bilevel problem, Annals of Operations Research, 181 (2010) 423-442.

[89] V.V. Kalashnikov, S. Dempe, G.A. Pérez-Valdés, N.I. Kalashnykova, J.-F. Camacho-Vallejo, Bilevel programming and applications, Mathematical Problems in Engineering, 2015 (2015) 1-16.

[90] C. Kasemset, V. Kachitvichyanukul, A PSO-based procedure for a bi-level multi-objective TOC-based job-shop scheduling problem, International Journal of Operational Research, 14 (2012) 50-69.

[91] T. Kis, A. Kovács, Exact solution approaches for bilevel lot-sizing, European Journal of Operational Research, 226 (2013) 237-245.

[92] D. Konur, M.M. Golias, Analysis of different approaches to cross-dock truck scheduling with truck arrival time uncertainty, Computers \& Industrial Engineering, 65 (2013) 663-672. 
[93] R.J. Kuo, Y.S. Han, A hybrid of genetic algorithm and particle swarm optimization for solving bi-level linear programming problem: A case study on supply chain model, Applied Mathematical Modelling, 35 (2011) 3905-3917.

[94] R.J. Kuo, C.C. Huang, Application of particle swarm optimization algorithm for solving bi-level linear programming problem, Computers \& Mathematics with Applications, 58 (2009) 678-685.

[95] Y.-J. Lai, Hierarchical optimization: A satisfactory solution, Fuzzy Sets and Systems, 77 (1996) 321-335.

[96] K.-M. Lan, U.-P. Wen, H.-S. Shih, E.S. Lee, A hybrid neural network approach to bilevel programming problems, Applied Mathematics Letters, 20 (2007) 880-884.

[97] D. Li, J.B. Cruz Jr, Information, decision-making and deception in games, Decision Support Systems, 47 (2009) 518-527.

[98] B. Liu, Stackelberg-Nash equilibrium for multilevel programming with multiple followers using genetic algorithms, Computers \& Mathematics with Applications, 36 (1998) 79-89.

[99] W. Liu, Z. Zheng, K.-Y. Cai, Bi-level programming based real-time path planning for unmanned aerial vehicles, Knowledge-Based Systems, 44 (2013) 34-47.

[100] J. Lu, C. Shi, An extended branch and bound algorithm for bilevel multi-follower decision making in a referential-uncooperative situation, International Journal of Information Technology and Decision Making, 6 (2007) 371-388.

[101] J. Lu, C. Shi, G. Zhang, On bilevel multi-follower decision making: General framework and solutions, Information Sciences, 176 (2006) 1607-1627.

[102] J. Lu, C. Shi, G. Zhang, T. Dillon, Model and extended Kuhn-Tucker approach for bilevel multi-follower decision making in a referential-uncooperative situation, Journal of Global Optimization, 38 (2007) 597-608.

[103] J. Lu, G. Zhang, J. Montero, L. Garmendia, Multifollower trilevel decision making models and system, IEEE Transactions on Industrial Informatics, 8 (2012) 974-985.

[104] Z. Lukač, K. Šorić, V.V. Rosenzweig, Production planning problem with sequence dependent setups as a bilevel programming problem, European Journal of Operational Research, 187 (2008) 1504-1512.

[105] N. Lv, X. Yan, K. Xu, C. Wu, Bi-level programming based contra flow optimization for evacuation events, Kybernetes, 39 (2010) 1227-1234.

[106] Y. Lv, Z. Chen, Z. Wan, A neural network for solving a convex quadratic bilevel programming problem, Journal of Computational and Applied Mathematics, 234 (2010) 505-511.

[107] Y. Lv, T. Hu, G. Wang, Z. Wan, A neural network approach for solving nonlinear bilevel programming problem, Computers \& Mathematics with Applications, 55 (2008) 2823-2829.

[108] W. Ma, M. Wang, X. Zhu, Improved particle swarm optimization based approach for bilevel programming problem: An application on supply chain model, International Journal of Machine Learning and Cybernetics, 5 (2014) 281-292.

[109] A. Mersha, S. Dempe, Direct search algorithm for bilevel programming problems, Computational Optimization and Applications, 49 (2011) 1-15.

[110] A. Mitsos, Global solution of nonlinear mixed-integer bilevel programs, Journal of Global Optimization, 47 (2010) 557-582.

[111] A. Mitsos, P. Lemonidis, P. Barton, Global solution of bilevel programs with a nonconvex inner program, Journal of Global Optimization, 42 (2008) 475-513.

[112] J.T. Moore, J.F. Bard, The mixed integer linear bilevel programming problem, Operations Research, 38 (1990) 911.

[113] P.-y. Nie, Dynamic discrete-time multi-leader-follower games with leaders in turn, Computers \& Mathematics with Applications, 61 (2011) 2039-2043.

[114] I. Nishizaki, M. Sakawa, Computational methods through genetic algorithms for obtaining Stackelberg solutions to two-level integer programming problems, Cybernetics and Systems, 36 (2005) 565-579.

[115] H. Önal, A modified simplex approach for solving bilevel linear programming problems, European Journal of Operational Research, 67 (1993) 126-135. 
[116] M.S. Osman, M.A. Abo-Sinna, A.H. Amer, O.E. Emam, A multi-level non-linear multi-objective decision-making under fuzziness, Applied Mathematics and Computation, 153 (2004) 239-252.

[117] F. Plastria, L. Vanhaverbeke, Discrete models for competitive location with foresight, Computers \& Operations Research, 35 (2008) 683-700.

[118] S. Pramanik, Bilevel programming problem with fuzzy parameters: A fuzzy goal programing approach, Journal of Applied Quantitative Methods, 7 (2012) 9-24.

[119] S. Pramanik, T.K. Roy, Fuzzy goal programming approach to multilevel programming problems, European Journal of Operational Research, 176 (2007) 1151-1166.

[120] X. Qiu, G.Q. Huang, Storage pricing, replenishment, and delivery schedules in a supply hub in industrial park: A bilevel programming approach, International Journal of Production Research, 51 (2013) 6950-6971.

[121] A. Ren, Y. Wang, A cutting plane method for bilevel linear programming with interval coefficients, Annals of Operations Research, 223 (2014) 355-378.

[122] G. Ren, Z. Huang, Y. Cheng, X. Zhao, Y. Zhang, An integrated model for evacuation routing and traffic signal optimization with background demand uncertainty, Journal of Advanced Transportation, 47 (2013) 4-27.

[123] M.J. Rider, J.M. López-Lezama, J. Contreras, A. Padilha-Feltrin, Bilevel approach for optimal location and contract pricing of distributed generation in radial distribution systems using mixed-integer linear programming, IET Generation, Transmission \& Distribution, 7 (2013) 724-734.

[124] G.Z. Ruan, S.Y. Wang, Y. Yamamoto, S.S. Zhu, Optimality conditions and geometric properties of a linear multilevel programming problem with dominated objective functions, Journal of Optimization Theory and Applications, 123 (2004) 409-429.

[125] M. Sakawa, I. Nishizaki, Interactive fuzzy programming for two-level nonconvex programming problems with fuzzy parameters through genetic algorithms, Fuzzy Sets and Systems, 127 (2002) 185-197.

[126] M. Sakawa, I. Nishizaki, Interactive fuzzy programming for multi-level programming problems: a review, International Journal of Multicriteria Decision Making, 2 (2012) 241-266.

[127] M. Sakawa, I. Nishizaki, M. Hitaka, Interactive fuzzy programming for multi-level 0-1 programming problems with fuzzy parameters through genetic algorithms, Fuzzy Sets and Systems, 117 (2001) 95-111.

[128] M. Sakawa, I. Nishizaki, Y. Uemura, Interactive fuzzy programming for multilevel linear programming problems, Computers \& Mathematics with Applications, 36 (1998) 71-86.

[129] M. Sakawa, I. Nishizaki, Y. Uemura, Interactive fuzzy programming for multi-level linear programming problems with fuzzy parameters, Fuzzy Sets and Systems, 109 (2000) 3-19.

[130] M. Sakawa, I. Nishizaki, Y. Uemura, Interactive fuzzy programming for two-level linear fractional programming problems with fuzzy parameters, Fuzzy Sets and Systems, 115 (2000) 93-103.

[131] S.S. Sana, A production-inventory model of imperfect quality products in a three-layer supply chain, Decision Support Systems, 50 (2011) 539-547.

[132] M.P. Scaparra, R.L. Church, A bilevel mixed-integer program for critical infrastructure protection planning, Computers \& Operations Research, 35 (2008) 1905-1923.

[133] H. Shao, W.H.K. Lam, A. Sumalee, A. Chen, M.L. Hazelton, Estimation of mean and covariance of peak hour origin-destination demands from day-to-day traffic counts, Transportation Research Part B: Methodological, 68 (2014) 52-75.

[134] V. Sharma, K. Dahiya, V. Verma, A class of integer linear fractional bilevel programming problems, Optimization, 63 (2014) 1565-1581.

[135] C. Shi, J. Lu, G. Zhang, An extended Kth-best approach for linear bilevel programming, Applied Mathematics and Computation, 164 (2005) 843-855.

[136] C. Shi, J. Lu, G. Zhang, H. Zhou, An extended branch and bound algorithm for linear bilevel programming, Applied Mathematics and Computation, 180 (2006) 529-537.

[137] C. Shi, G. Zhang, J. Lu, The Kth-Best approach for linear bilevel multi-follower programming, Journal of Global 
Optimization, 33 (2005) 563-578.

[138] C. Shi, H. Zhou, J. Lu, G. Zhang, Z. Zhang, The Kth-best approach for linear bilevel multifollower programming with partial shared variables among followers, Applied Mathematics and Computation, 188 (2007) 1686-1698.

[139] H.-S. Shih, Y.-J. Lai, E.S. Lee, Fuzzy approach for multi-level programming problems, Computers \& Operations Research, 23 (1996) 73-91.

[140] H.s. Shih, C.b. Cheng, U.p. Wen, Y.c. Huang, M.y. Peng, Determining a subsidy rate for Taiwan's recycling glass industry: An application of bi-level programming, The Journal of the Operational Research Society, 63 (2012) 28-37.

[141] A. Sinha, P. Malo, K. Deb, Test problem construction for single-objective bilevel optimization, Evolutionary Computation, 22 (2014) 439-477.

[142] A. Sinha, P. Malo, A. Frantsev, K. Deb, Finding optimal strategies in a multi-period multi-leader-follower Stackelberg game using an evolutionary algorithm, Computers \& Operations Research, 41 (2014) 374-385.

[143] S. Sinha, A comment on Anandalingam (1988). A mathematical programming model of decentralized multi-level systems. J Opl Res Soc 39: 1021-1033, Journal of the Operational Research Society, 52 (2001) 594-596.

[144] S. Sinha, Fuzzy mathematical programming applied to multi-level programming problems, Computers \& Operations Research, 30 (2003) 1259-1268.

[145] S. Sinha, Fuzzy programming approach to multi-level programming problems, Fuzzy Sets and Systems, 136 (2003) 189-202.

[146] O. Skulovich, L. Perelman, A. Ostfeld, Bi-level optimization of closed surge tanks placement and sizing in water distribution system subjected to transient events, Procedia Engineering, 89 (2014) 1329-1335.

[147] H.V. Stackelberg, The Theory of Market Economy, Oxford University Press, Oxford, 1952.

[148] A. Street, A. Moreira, J.M. Arroyo, Energy and reserve scheduling under a joint generation and transmission security criterion: An adjustable robust optimization approach, IEEE Transactions on Power Systems, 29 (2014) 3-14.

[149] A.F. Taha, N.A. Hachem, J.H. Panchal, A Quasi-Feed-In-Tariff policy formulation in micro-grids: A bi-level multi-period approach, Energy Policy, 71 (2014) 63-75.

[150] R.R. Tan, K.B. Aviso, J.B. Cruz Jr, A.B. Culaba, A note on an extended fuzzy bi-level optimization approach for water exchange in eco-industrial parks with hub topology, Process Safety \& Environmental Protection: Transactions of the Institution of Chemical Engineers Part B, 89 (2011) 106-111.

[151] H. Tuy, A. Migdalas, N.T. Hoai-Phuong, A novel approach to bilevel nonlinear programming, Journal of Global Optimization, 38 (2007) 527-554.

[152] H. Tuy, A. Migdalas, P. Värbrand, A global optimization approach for the linear two-level program, Journal of Global Optimization, 3 (1993) 1-23.

[153] S. Ukkusuri, K. Doan, H.M.A. Aziz, A bi-level formulation for the combined dynamic equilibrium based traffic signal control, Procedia - Social and Behavioral Sciences, 80 (2013) 729-752.

[154] L. Vicente, P. Calamai, Bilevel and multilevel programming: A bibliography review, Journal of Global Optimization, 5 (1994) 291-306.

[155] L. Vicente, G. Savard, J. Judice, Discrete linear bilevel programming problem, Journal of Optimization Theory and Applications, 89 (1996) 597-614.

[156] Z. Wan, L. Mao, G. Wang, Estimation of distribution algorithm for a class of nonlinear bilevel programming problems, Information Sciences, 256 (2014) 184-196.

[157] Z. Wan, G. Wang, B. Sun, A hybrid intelligent algorithm by combining particle swarm optimization with chaos searching technique for solving nonlinear bilevel programming problems, Swarm and Evolutionary Computation, 8 (2013) 26-32.

[158] S. Wang, Q. Meng, H. Yang, Global optimization methods for the discrete network design problem, Transportation Research Part B: Methodological, 50 (2013) 42-60.

[159] Y. Wang, Y.-C. Jiao, H. Li, An evolutionary algorithm for solving nonlinear bilevel programming based on a new constraint-handling scheme, IEEE Transactions on Systems, Man, and Cybernetics, Part C: Applications and Reviews, 35 
(2005) 221-232.

[160] U.P. Wen, A.D. Huang, A simple Tabu Search method to solve the mixed-integer linear bilevel programming problem, European Journal of Operational Research, 88 (1996) 563-571.

[161] U.P. Wen, Y.H. Yang, Algorithms for solving the mixed integer two-level linear programming problem, Computers \& Operations Research, 17 (1990) 133-142.

[162] D.J. White, Penalty function approach to linear trilevel programming, Journal of Optimization Theory and Applications, 93 (1997) 183-197.

[163] D.J. White, G. Anandalingam, A penalty function approach for solving bi-level linear programs, Journal of Global Optimization, 3 (1993) 397-419.

[164] J. Xu, J. Gang, Multi-objective bilevel construction material transportation scheduling in large-scale construction projects under a fuzzy random environment, Transportation Planning and Technology, 36 (2013) 352-376.

[165] P. Xu, L. Wang, An exact algorithm for the bilevel mixed integer linear programming problem under three simplifying assumptions, Computers \& Operations Research, 41 (2014) 309-318.

[166] X. Xu, Z. Meng, R. Shen, A tri-level programming model based on Conditional Value-at-Risk for three-stage supply chain management, Computers \& Industrial Engineering, 66 (2013) 470-475.

[167] D. Yang, J. Jiao, Y. Ji, G. Du, P. Helo, A. Valente, Joint optimization for coordinated configuration of product families and supply chains by a leader-follower Stackelberg game, European Journal of Operational Research, In Press (2015).

[168] Y. Yao, T. Edmunds, D. Papageorgiou, R. Alvarez, Trilevel optimization in power network defense, IEEE Transactions on Systems, Man, and Cybernetics, 37 (2007) 712-718.

[169] G. Zhang, J. Lu, The definition of optimal solution and an extended Kuhn-Tucker approach for fuzzy linear bi-level programming, IEEE Computational Intelligence Bulletin, 2 (2005) 1-7.

[170] G. Zhang, J. Lu, Model and approach of fuzzy bi-level decision making for logistics planning problem, Journal of Enterprise Information Management, 20 (2007) 178-197.

[171] G. Zhang, J. Lu, Fuzzy bilevel programming with multiple objectives and cooperative multiple followers, Journal of Global Optimization, 47 (2010) 403-419.

[172] G. Zhang, J. Lu, T. Dillon, Decentralized multi-objective bilevel decision making with fuzzy demands, Knowledge-Based Systems, 20 (2007) 495-507.

[173] G. Zhang, J. Lu, T. Dillon, Models and algorithm for fuzzy multi-objective multi-follower linear bilevel programming, in: IEEE International Fuzzy Systems Conference, 2007, pp. 1-6.

[174] G. Zhang, J. Lu, Y. Gao, An algorithm for fuzzy multi-objective multi-follower partial cooperative bilevel programming, Journal of Intelligent \& Fuzzy Systems, 19 (2008) 303-319.

[175] G. Zhang, J. Lu, Y. Gao, Multi-Level Decision Making: Models, Methods and Applications, Springer, Berlin, 2015.

[176] G. Zhang, J. Lu, J. Montero, Y. Zeng, Model, Solution concept and the Kth-best algorithm for linear tri-level programming, Information Sciences, 180 (2010) 481-492.

[177] G. Zhang, G. Zhang, Y. Gao, J. Lu, A bilevel optimization model and a PSO-based algorithm in day-ahead electricity markets, in: IEEE International Conference on Systems, Man and Cybernetics, San Antonio, TX, 2009, pp. 617-622.

[178] G. Zhang, G. Zhang, Y. Gao, J. Lu, Competitive strategic bidding optimization in electricity markets using bilevel programming and swarm technique, IEEE Transactions on Industrial Electronics, 58 (2011) 2138-2146.

[179] T. Zhang, T. Hu, X. Guo, Z. Chen, Y. Zheng, Solving high dimensional bilevel multiobjective programming problem using a hybrid particle swarm optimization algorithm with crossover operator, Knowledge-Based Systems, 53 (2013) 13-19. 